\title{
Inefficiency and complementarity in sharing games
}

\author{
Dennis Courtney • Thomas Marschak
}

Received: 9 October 2008 / Accepted: 27 November 2008 / Published online: 30 December 2008 (C) The Author(s) 2008. This article is published with open access at Springerlink.com

\begin{abstract}
A sharing game is a very simple device for partially reconciling an organization's goal with the interests of its members. Each member chooses an action, bears its cost, and receives a share of the revenue which the members' actions generate. A (pure-strategy) equilibrium of the game may be inefficient: surplus (revenue minus the sum of costs) may be less than maximal. In a previous paper, we found that for a wide class of reward functions, no one squanders at an inefficient equilibrium (spends more than at an efficient profile) if the revenue function has a complementarity property. In the present paper, we examine the "opposite" of the complementarity property (Substitutes) and we study a class of finite games where squandering equilibria indeed occur if Substitutes holds strongly enough. Squandering equilibria play a key role when one traces the effect of technological improvement on a sharing game's surplus shortfall. We then turn to the question of choice among reward functions in a principal/agents setting. We find that if we again assume complementarity then strong conclusions can be reached about the reward functions preferred by "society", by the players (agents), and by the principal.
\end{abstract}

Keywords Organizations · Decentralization · Sharing games · Complementarity · Efficiency

JEL Classification $\quad$ C70 $\cdot$ D20 $\cdot$ D80

D. Courtney

Department of Mathematics, University of Iowa, Iowa City, USA

T. Marschak $(\varangle)$

Walter A. Haas School of Business, University of California, Berkeley, USA

e-mail:marschak@haas.berkeley.edu 


\section{Introduction}

A sharing game is a simple device for an organization which has a goal but consists of $n$ self-interested members. Each member chooses an action (strategy) from a set of possible actions and bears that action's cost. The $n$ actions determine a revenue. The game's designer chooses a reward function for each member, defined on the possible revenues. We then have a game in which, for a given action profile, each player's payoff is his reward minus his cost. The informational requirements of the arrangement are very modest, since no messages are sent. Truth-telling is not an issue. There is no monitoring. It is hardly surprising that in the real world, sharing schemes are among the oldest and most common arrangements for partially reconciling organizational goals with individual incentives.

Suppose, moreover, that one seeks to understand how well "decentralization", with respect to information and with respect to authority, performs relative to "centralization". Two extreme benchmarks are useful. In the extreme centralized structure, a center has full access to members' private information and can command their choices. A sharing game is a natural candidate for the extreme decentralized opposite of that structure. Members do what they want, no one sees their choices, and no one asks about their private information.

Of course a sharing game's informational and authority-free virtues may be offset by a penalty: the actions chosen at an equilibrium of the game may not be efficient. They may not maximize surplus, i.e. revenue minus total costs. To understand the penalty (the "price of decentralizing"), a general theory of sharing games is needed.

In Courtney and Marschak (2006), an earlier paper, we developed some of the fundamentals of a general theory. We found that complementarity in the revenue function yields strong conclusions. In particular, we found that if we have complementarity then, for a wide class of reward functions and any increasing cost functions, there cannot be an equilibrium at which some player squanders, i.e. spends more (or "works harder") than he does in an efficient strategy profile. In the present paper, we continue the task. We explore the following two questions: (1) When does the "opposite" of complementarity (the "substitutes" property) imply that at some equilibria squandering indeed occurs? (2) Given a simple class of reward functions (e.g., constant-share functions) which members of that class yield high surplus at the game's equilibria?

\subsection{The framework}

In an $n$-player sharing game, player $i$ chooses a strategy or action $x_{i}$ from a set $S_{i}$. Those choices yield a revenue $A(x)$, where $x$ denotes the strategy (action) profile $\left(x_{1}, \ldots, x_{n}\right)$ and $A$ is a function from $S \equiv S_{1} \times \cdots \times S_{n}$ to the nonnegative real numbers. When player $i$ chooses the strategy $x_{i}$ he pays the nonnegative cost $c_{i}\left(x_{i}\right)$. It is often helpful to use the term effort instead of "cost" and we use the two terms interchangeably. Once $x$ is chosen and the resulting revenue is realized, Player $i$ receives the reward $\rho_{i}(A(x))$, where $\rho_{i}$ is a function from $A(S)$, the set of possible revenues, to the nonnegative real numbers. Player $i$ 's net payoff at the profile $x$ is $\rho_{i}(A(x))-c_{i}\left(x_{i}\right)$. Letting $N$ denote the player set $\{1, \ldots, n\}$, the game is defined by the quadruple $\left\langle\left\{S_{i}\right\}_{i \in N}, A,\left\{\rho_{i}\right\}_{i \in N},\left\{c_{i}\right\}_{i \in N}\right\rangle$. 
Note that a player may have several equally costly strategies. The literature, by contrast, usually confines attention to games in which a strategy is uniquely determined by its cost or effort level. We shall call such games effort games. ${ }^{1}$ We confine attention to a sharing game's pure-strategy equilibria. We will be interested in the surplus at each equilibrium. The surplus at a profile $x$ is $A(x)-\tau(x)$, where $\tau(x)$ denotes the total cost $\sum_{i \in N} c_{i}\left(x_{i}\right)$. A profile which maximizes surplus is efficient. A profile $x$ is squandering (shirking) relative to an efficient profile $y$ if $\tau(x)>\tau(y)(\tau(x)<\tau(y))$.

While our formal framework is stated in deterministic style, it nevertheless covers probabilistic settings if the players are risk neutral and the reward functions are linearly related. ${ }^{2}$

\subsection{Organization of the paper}

In Sect. 2, we consider two sharing-game settings: a principal with agents and a budget-balancing partnership. ${ }^{3}$ We then introduce a broad class of reward functions. They are nondecreasing and they have the "nondecreasing residual" (NDR) property. Those properties can be justified, under certain assumptions, for each of our two settings. We summarize some of our previous results on existence of equilibrium and on equilibria at which squandering occurs. In Sect. 3, we provide a strong motivation for studying the squandering phenomenon, namely the impact of technological improvement on organizational structure. Whether or not technological improvement strengthens the appeal of sharing games relative to the centralized structure depends on the type of sharing-game equilibria one considers. The effect of technological improvement on the surplus shortfall at a squandering equilibrium is very different than the effect for

\footnotetext{
1 The framework just given, in its effort-game version, fits a number of papers, starting with Holmstrom (1982). Recent papers include Arya et al. (1997), Che and Yoo (2001), Ishida (2006), Legros and Matthews (1993), Levitt (1995) and Nandeibam (2002).

${ }^{2}$ First suppose there is a random variable $\theta$, whose probability distribution is common knowledge. Revenue depends on that variable's value as well as the chosen strategies, and is written as $A(x, \theta)$. If players are risk-neutral, then Player $i$ 's net payoff is the expected value of $\rho_{i}(A(x, \theta))-c_{i}\left(x_{i}\right)$. Suppose the reward functions are linearly related; for all $i$, we have $\rho_{i}=k_{i} \cdot \rho$, where $k_{i}>0$. If we now define $\tilde{A}(x)$ to be the expected value of $\rho(A(x, \theta))$, then we can write $i$ 's net payoff for the profile $x$ as $\tilde{\rho}_{i}(\tilde{A}(x))-c_{i}\left(x_{i}\right)$, where $\tilde{\rho}_{i}(z)=k_{i} \cdot z$. So the game can be rewritten as the deterministic game defined by $\tilde{A}$, the reward functions $\tilde{\rho}_{i}$, and the original strategy sets $S_{i}$ and cost functions $c_{i}$.

Next, we can introduce uncertainty about individual costs in addition to the uncertainty just considered, while still using our formalism. Interpret the strategies $x_{i}$, the sets $S_{i}$ and the functions $A$ and $c_{i}$ in the following way. Player $i$ repeatedly and privately observes a random signal $e_{i}$ whose values lie in a set $E_{i}$. The random variable $e_{i}$ influences $i$ 's costs and in addition the $n$-tuple $e=\left(e_{1}, \ldots, e_{n}\right)$, whose probability distribution is common knowledge, influences revenue. Player $i$ responds to the signal $e_{i}$ with an action $b_{i}$ in a set $B_{i}$, where $b_{i} \operatorname{costs} \bar{c}_{i}\left(b_{i}, e_{i}\right)$. To find that action, $i$ uses a response rule $x_{i}: E_{i} \rightarrow B_{i}$. The response rule $x_{i}$ is chosen, once and for all, from a set $S_{i}$ of admissible response rules. For a given value of $\left(e_{1}, \ldots, e_{n}\right)$, the action $n$-tuple $\left(b_{1}, \ldots, b_{n}\right)$ yields the revenue $\alpha\left(\left(b_{1}, \ldots, b_{n}\right), e\right)$ and Player $i$ receives the reward $k_{i} \cdot \rho\left(\alpha\left(\left(b_{1}, \ldots, b_{n}\right), e\right)\right)$. Now let $A\left(x_{1}, \ldots, x_{n}\right)$ denote the expected value of $\rho\left(\alpha\left(\left(x_{1}\left(e_{1}\right), \ldots, x_{n}\left(e_{n}\right)\right), e\right)\right)$. Then $i$ 's net payoff for any $x=\left(x_{1}, \ldots, x_{n}\right)$ is the expected value of his reward minus his expected cost, i.e., it is $k_{i} \cdot A\left(x_{1}, \ldots, x_{n}\right)-c_{i}\left(x_{i}\right)$, where $c_{i}\left(x_{i}\right)$ denotes the expected value of $\bar{c}_{i}\left(x_{i}\left(e_{i}\right), e_{i}\right)$.

3 The discussion of these two settings is much more detailed than the very brief remarks made in Courtney and Marschak (2006).
} 
a shirking equilibrium. In Sect. 4, we study the implications of a sufficiently strong Substitutes property for the existence of squandering equilibria in a class of finite two-player games. In Sect. 5, we briefly consider three information-gathering games, in which each player specializes in a private random variable and learns more about its current value by spending more. Revenue is earned when each player reports what he has learned to Headquarters, which then takes an appropriate action. It is plausible that squandering ("information overload") may occur at the game's equilibria, but a great deal remains to be learned. In Sect. 6, we turn to the choice of reward functions, and the associated surplus. We find that surplus increases when we move from an equilibrium of any given sharing game to an equilibrium of an NDR game, if the players work harder at the latter equilibrium. We find that if two games differ with regard to reward functions only, players prefer an equilibrium of the first game to an equilibrium of the second if they work harder at the first equilibrium, provided the reward functions in the first game are more generous. We then turn to a key question: when do more generous rewards indeed imply a harder-working equilibrium (and hence higher surplus)? Here complementarity plays a crucial role. We apply the findings to bargaining between principal and players (agents) as to the constant-share reward functions that will be used. We find, for selected equilibria, that the more weight is given to the players' preferences in choosing the reward functions, the harder players work and hence the higher is surplus. In Sect. 7, we make some summarizing remarks.

\section{Two settings, and a class of reward functions}

\subsection{Two settings: a principal with agents and a partnership}

Suppose the organization consists of a principal and his agents. The strategy sets, revenue function and cost functions are given. The principal designs the game by choosing the reward functions and the agents agree to play it. After they choose their strategies, the principal pays them their rewards and pockets the residual, i.e., the realized revenue minus the rewards. The principal seeks to design a game in which residual is maximal at the game's equilibria. But the principal's design choices are constrained because there is an exogenously given Participation Constraint (PC). Consider the PC that is defined when each agent $i$ states

A necessary condition for my participation in the game is that at every (purestrategy) equilibrium of the game my net payoff be at least $J_{i}$, i.e.,

$$
\text { if } x \text { is an equilibrium, then } \rho_{i}(A(x)) \geq J_{i}+c_{i}\left(x_{i}\right) \text {. }
$$

The number $J_{i}$ reflects the other options available to player $i$. Now suppose that $x^{*}=\left(x_{1}^{*}, \ldots, x_{n}^{*}\right)$ is efficient for a given triple $\left\langle\left\{S_{i}\right\}_{i \in N}, A,\left\{c_{i}\right\}_{i \in N}\right\rangle$, i.e.,

$$
\text { for all } x \in S, A\left(x^{*}\right)-\tau\left(x^{*}\right) \geq A(x)-\tau(x) \text {. }
$$

Then

if the sharing game $G$ satisfies the PC, an upper bound to the principal's residual at any (pure-strategy) equilibrium of the game is $A\left(x^{*}\right)-\tau\left(x^{*}\right)-\sum_{i \in N} J_{i}$. 
That is the case since for any equilibrium $\bar{x} \in S$ we have, using (+) and (++),

$$
A(\bar{x})-\sum_{i \in N} \rho_{i}(A(\bar{x})) \leq A(\bar{x})-\tau(\bar{x})-\sum_{i \in N} J_{i} \leq A\left(x^{*}\right)-\tau\left(x^{*}\right)-\sum_{i \in N} J_{i} .
$$

Next suppose that the set $S=S_{1} \times \cdots \times S_{n}$ contains at least one minimal-cost strategy profile, say $\tilde{x}=\left(\tilde{x}_{1}, \ldots, \tilde{x}_{n}\right)$, i.e., for all $i \in N$ and for all $x_{i} \in S_{i}$, we have $c_{i}\left(\tilde{x}_{i}\right) \leq c_{i}\left(x_{i}\right)$. Then the following "collective punishment" reward functions $\rho_{i}$, which are prominent in the literature, may appeal to a residual-maximizing principal:

$$
\rho_{i}(z)= \begin{cases}J_{i}+c_{i}\left(x_{i}^{*}\right)+\delta & \text { if } z=A\left(x^{*}\right) \\ J_{i}+c_{i}\left(\tilde{x}_{i}\right) & \text { if } z \neq A\left(x^{*}\right)\end{cases}
$$

where $\delta>0$. The efficient profile $x^{*}$ is an equilibrium of this game, and so is any other efficient profile $x$ for which $A(x)=A\left(x^{*}\right)$ and $c_{i}\left(x_{i}\right)=c_{i}\left(x^{*}\right)$ for all $i .{ }^{4}$ Moreover any profile $x$ for which $A(x) \neq A\left(x^{*}\right)$ and some player $i$ 's cost is not minimal cannot be an equilibrium, because at that profile, $i$ receives the smallest possible reward, and would continue to receive it, while increasing his net payoff, if he deviates to a strategy at which his cost is minimal. A profile like $\tilde{x}$, at which every player's cost is minimal, may also be an equilibrium, ${ }^{5}$ but it is Pareto-dominated (at least weakly) by the equilibrium $x^{*}$. We may, moreover, have a "free-riding" equilibrium, say $x^{\prime}$, where $A\left(x^{\prime}\right)=A\left(x^{*}\right)$ but for some players cost exceeds cost at $x^{*}$ while for other players the reverse is true. ${ }^{6}$

To summarize, the strongest statement that can be made in favor of the collectivepunisment reward functions is as follows.

By making $\delta$ positive and sufficiently small, we can ensure that for the collectivepunishment game: (1) the PC is met, (2) the efficient profiles are equilibria and they Pareto-dominate the minimal-cost equilibria, (3) the residual at an efficient equilibrium is as close as desired to the upper bound on residual. ${ }^{7}$

\footnotetext{
4 If Player $i$ unilaterally deviates from $x_{i}^{*}$ to another strategy which costs the same as $x_{i}^{*}$, then he continues to get the net payoff $J_{i}+c_{i}\left(x_{i}^{*}\right)+\delta$ if the resulting new profile again yields the revenue $A\left(x^{*}\right)$, and a lower payoff if it does not. If he deviates to a costlier strategy (whether or not the new profile yields $A\left(x^{*}\right)$ ), then his net payoff is smaller than it was, since his reward cannot exceed $J_{i}+c_{i}\left(x_{i}^{*}\right)+\delta$. If he deviates to a cheaper strategy, say $\bar{x}_{i}$, then the resulting revenue cannot equal $A\left(x^{*}\right)$ (if it did, then $x^{*}$ would not be efficient) and hence his net payoff would shrink to $J_{i}+c_{i}\left(\tilde{x}_{i}\right)-c_{i}\left(\bar{x}_{i}\right)$.

5 That is the case, in particular, if any profile at which some player's cost is minimal yields a revenue that does not equal $A\left(x^{*}\right)$. Then a player gains nothing by deviating from $\tilde{x}_{i}$ to a costlier strategy, for then revenue remains distinct from $A\left(x^{*}\right)$ and so his net payoff shrinks. He cannot deviate to a cheaper strategy because there are none, and deviating to an equally costly stategy leaves his payoff unchanged.

6 At such an equilibrium $x^{\prime}$, all players receive the highest possible reward. Clearly no player can gain by deviating from $x^{\prime}$ to a costlier strategy. Suppose it is the case that every unilateral deviation from $x^{\prime}$ to a lower-cost strategy produces a revenue distinct from $A\left(x^{*}\right)$ and hence yields the lowest possible reward. If the cost saving for a player who deviates from $x^{\prime}$ to a cheaper strategy is never more than the reward reduction, then no player gains from a deviation to a cheaper strategy.

7 The principal's residual at $x^{*}$ is $A\left(x^{*}\right)-\sum_{i \in N} J_{i}-\tau\left(x^{*}\right)-n \delta$. For $\delta=0$ that equals the upper bound given above. Parts of the preceding discussion can be found (in a somewhat different form) in Mookherjee's
} 
Some might conclude from the preceding three-part statement, that the matter of choosing reward functions is "closed", i.e., there is no reason for the principal to have the slightest interest in reward functions other than the collective-punishment functions, so the study of other reward functions is unmotivated and superfluous. We would disagree. First, note that the reward-function designer needs to know enough about the cost functions $c_{i}$ so that he can compute $A\left(x^{*}\right)$ and the quantities $c_{i}\left(x^{*}\right)$. Other reward functions (e.g., constant-share functions) require no such knowledge. Next, note that the PC is a necessary condition for the agents to agree to become players, but it is not sufficient. Among the many games which satisfy the PC, some are more attractive to the agents than others, and the same is true for the principal. The collective-punishment game has a knife-edge property: small deviations from $x^{*}$ are punished just as severely as large deviations. The agents, and perhaps the principal, may find this unpleasant. The possibility of undominated inefficient free-riding equilibria is unattractive as well. ${ }^{8}$

Now consider partnerships. The players share the revenue and none of it is thrown away. Thus the admissible reward functions satisfy the balanced-budget condition $\sum_{i \in N} \rho_{i}(z)=z$. It is again appropriate to impose a participation constraint, say the above PC. The partners seek a reward-function $n$-tuple such that in the equilibria of the resulting game, $A(x)-\tau(x)$ (the surplus that they are dividing) is maximal. Within the set of such reward functions a reward-function $n$-tuple is selected, perhaps by bargaining among the $n$ partners. If $A$, the $\rho_{i}$, and the $c_{i}$ are differentiable, then, as Holmstrom first pointed out, an equilibrium of the game is never a maximizer of $A-\tau .{ }^{9}$ For finite games, the situation is different. It is easy to construct plausible finite

\section{Footnote 7 continued}

pioneering version (Mookherjee 1984) of the problem facing a residual-maximizing principal with many agents. A number of later writings varied Mookherjee's problem.

8 The following two-player example is instructive. $S_{1}=S_{2}=[0,1], A=x_{1}+x_{2}, c_{i}\left(x_{i}\right)=x_{i}^{2}$, and $J_{1}=J_{2}=0$. The unique efficient profile is $\left(\frac{1}{2}, \frac{1}{2}\right)$, which yields the revenue 1 . Let $i$ 's reward function be $\rho_{i}(z)=\left\{\begin{array}{ll}\frac{1}{4}+\delta & \text { if } z=1 \\ 0 & \text { if } z \neq 1\end{array}\right.$, where $\delta>0$. Then $\left(\frac{1}{2}, \frac{1}{2}\right)$ is an equilibrium, but so is $\left(\frac{1}{2}+\delta^{\prime}, \frac{1}{2}-\delta^{\prime}\right)$ (which also yields the revenue 1), provided $\delta^{\prime}$ is positive and satisfies $\delta>\delta^{\prime}+\left(\delta^{\prime}\right)^{2}$. A positive $\delta^{\prime}$ satisfying that inequality exists no matter how small $\delta$ may be. In the absence of deviation, Player 1's net payoff is $\frac{1}{4}+\delta-\left(\frac{1}{2}+\delta^{\prime}\right)^{2}=\delta-\delta^{\prime}-\left(\delta^{\prime}\right)^{2}$ and 2's net payoff is $\frac{1}{4}+\delta-\left(\frac{1}{2}-\delta^{\prime}\right)^{2}=\delta+\delta^{\prime}-\left(\delta^{\prime}\right)^{2}$. Since $\delta>\delta^{\prime}+\left(\delta^{\prime}\right)^{2}$, each player's net payoff is positive. If either player deviates from $\left(\frac{1}{2}+\delta^{\prime}, \frac{1}{2}-\delta^{\prime}\right)$, then revenue differs from 1 and hence each player's net payoff is zero minus his cost. So a deviation lowers his payoff. At the equilibrium $\left(\frac{1}{2}+\delta^{\prime}, \frac{1}{2}-\delta^{\prime}\right)$, Player 2 is the free rider. The free-riding equilibrium is not Pareto-dominated by the efficient equilibrium. Note that total cost at the free-riding equilibrium is $\left(\frac{1}{2}+\delta^{\prime}\right)^{2}+\left(\frac{1}{2}-\delta^{\prime}\right)^{2}=\frac{1}{2}+2\left(\delta^{\prime}\right)^{2}$, while total cost at $\left(\frac{1}{2}, \frac{1}{2}\right)$ is $\frac{1}{2}$. So the free-riding equilibrium is squandering relative to the efficient profile.

9 This follows, in fact, from a weaker condition than budget balancing. It is enough to require that for every $z \in A(S)$, there is some player, say $i(z)$, for whom $\rho_{i(z)}^{\prime}(z) \neq 1$. The surplus-maximization first-order condition is $A_{i}(x)=c_{i}^{\prime}\left(x_{i}\right)$, all $i$ (where $A_{i}$ denotes the derivative of $A$ with respect to $x_{i}$ ). The first-order condition for an equilibrium of the sharing game is $\rho_{i}^{\prime}(A(x)) \cdot A_{i}(x)=c_{i}^{\prime}\left(x_{i}\right)$, all $i$. Thus if $x^{\#} \in A(S)$ satisfies both the surplus-maximization condition and the equuilibrium condition, then $\rho_{i}^{\prime}\left(A\left(x^{\#}\right)\right)=1$ for all $i$. That violates our requirement. 
budget-balancing sharing games where the only equilibrium is efficient, ${ }^{10}$ as well as finite games with several equilibria, with one or more of them efficient.

\subsection{A wide class of plausible reward functions}

Our criticism of collective-punishment reward functions was informal. We shall not propose a formal "acceptability" criterion for reward functions. But if we are to make progress, we have to impose some restrictions on the reward functions to be studied.

First we confine attention to nondecreasing reward functions. Second, in many of the results in Courtney and Marschak (2006) we confine attention to the wide class of Nondecreasing Residual (NDR) reward functions. For a given revenue $z$, the residual is $z-\sum_{i \in N} \rho_{i}(z)$. The reward-function profile $\left\{\rho_{i}\right\}_{i \in N}$ is said to have the NDR property if and only if the residual is nondecreasing in the revenue $z$. Balanced-budget functions have the NDR property, since the residual is always zero for those functions, and so do constant-share functions with shares summing to one or less $\left(\rho_{i}(z)=r_{i} \cdot z\right.$, where $\left.r_{i}>0, \sum_{i \in N} r_{i} \leq 1\right)$. Note that an NDR non-decreasing reward-function profile lies in a larger class, namely the class of profiles in which each function is nondecreasing and regressive. Player $i$ 's reward function $\rho_{i}$ is said to have that property if and only if $i$ 's reward never goes up by more than revenue, i.e.,

for all $z, \Delta$ with $\Delta>0$ and $z, z+\Delta \in A(S)$, we have $\rho_{i}(z+\Delta)-\rho_{i}(z) \leq \Delta$.

$\overline{10}$ Here is a symmetric two-player example. Let $n=2$. Let $S_{1}=S_{2}=\{0,1,2\}$. Let the revenue function $A$ be given by the following matrix

0
1
2 $\left(\begin{array}{ccc}0 & 1 & 2 \\ 14 & 35 & 27 \\ 27 & 39 & 42\end{array}\right)$

Let $c_{i}\left(x_{i}\right)=5 x_{i}, i=1,2$. Note that we have "diminishing marginal product": for a given choice of one player, the revenue increment declines as the other player increases effort. Surplus is given by:

0
1
2 $\left(\begin{array}{ccc}0 & 1 & 2 \\ 9 & 25 & 17 \\ 17 & 24 & 22\end{array}\right)$

The unique efficient pair is $(1,1)$. Let $\rho_{i}(z)=\frac{1}{2} z, i=1,2$. Then the payoff matrix of the sharing game is

$\begin{aligned} & 0 \\ & 1 \\ & 2\end{aligned}\left(\begin{array}{ccc} & 1 & 2 \\ (0,0) & (7,2) & (13.5,3.5) \\ (2,7) & (\mathbf{1 2 . 5}, \mathbf{1 2 . 5}) & (14.5,9.5) \\ (3.5,13.5) & (9.5,14.5) & (11,11)\end{array}\right)$.

The only equilibrium is the efficient pair $(1,1)$. 
If, in violation of regressivity, some player's reward went up by more than revenue, then the residual would have to go down. Note that if $\rho_{i}$ is differentiable, then regressivity implies that $\rho_{i}^{\prime} \leq 1$.

Observe that collective-punishment reward functions generally violate the NDR condition. At a revenue that is $\epsilon$ below the critical efficient revenue, the $n$ players' rewards are minimal. They jump to their highest level as soon as revenue reaches the critical value. If $\epsilon$ is small, the sum of the $n$ jumps exceeds the revenue increase, so that residual goes down.

Informally speaking, the nondecreasing and regressivity requirements on the reward functions appear to be rather weak. The nondecreasing property can be justified for the principal/agents setting in the following way. Call the pair $\left(A,\left\{c_{i}\right\}_{i \in N}\right)$ productive if one player's switch to a costlier strategy cannot decrease revenue, i.e., (using some standard notation):

for all $i \in N, \quad$ all $x_{i}, x_{i}^{\prime} \in S_{i}, \quad$ and all $x_{-i} \in S_{-i}, \quad c_{i}\left(x_{i}^{\prime}\right)>c_{i}\left(x_{i}\right) \Longrightarrow A\left(x_{i}^{\prime}, x_{-i}\right)$ $\geq A\left(x_{i}, x_{-i}\right)$.

If the second inequality is strict we shall call the pair $\left(A,\left\{c_{i}\right\}_{i \in N}\right)$ strictly productive. A sharing game $\left\langle\left\{S_{i}\right\}_{i \in N}, A,\left\{\rho_{i}\right\}_{i \in N},\left\{c_{i}\right\}_{i \in N}\right\rangle$ will be called productive (strictly productive) if the pair $\left(A,\left\{c_{i}\right\}_{i \in N}\right)$ is productive (strictly productive). Now suppose the game $\left\langle\left\{S_{i}\right\}_{i \in N}, A,\left\{\rho_{i}\right\}_{i \in N},\left\{c_{i}\right\}_{i \in N}\right\rangle$ is productive, all the sets $S_{i}$ are finite, and for the function $\rho_{1}$ (for example), for some $x_{-1} \in S_{-1}$, and some $x_{1}, x_{1}^{\prime} \in S_{1}$, we have (i) $c_{1}\left(x_{1}^{\prime}\right)>c_{1}\left(x_{1}\right)$, (ii) $A\left(x_{1}^{\prime}, x_{-1}\right)>A\left(x_{1}, x_{-1}\right)$, but (iii) $\rho_{1}\left(A\left(x_{1}^{\prime}, x_{-1}\right)<\right.$ $\rho_{1}\left(A\left(x_{1}, x_{-1}\right)\right)$. Note that $\left(x_{1}^{\prime}, x_{-1}\right)$ cannot be an equilibrium, since 1 will want to defect to $x_{1}$, where her cost is lower and her reward is higher. Now replace the reward function $\rho_{1}$ by a new function $\hat{\rho}_{1}$ which coincides with $\rho_{1}$ at all revenues except $A\left(x_{1}^{\prime}, x_{-1}\right)$; there we have $\hat{\rho}_{1}\left(A\left(x_{1}^{\prime}, x_{-1}\right)\right)=\rho_{1}\left(A\left(x_{1}, x_{-1}\right)\right)$. It is straightforward to check, using productivity, that this change does not eliminate any equilibria of the original game. In the partnership setting the change in $\rho_{1}$ has to be accompanied by compensating changes in the other $\rho_{i}$, so as to preserve budget-balancing. But again, none of the previous equilibria are eliminated. Sucessive corrections of this sort yield a new game with nondecreasing reward functions and with all equilibria of the original game preserved. ${ }^{11}$ Thus neither the principal's maximal equilibrium residual, nor the partners' maximal equilibrium surplus declines when we replace the old game by the new one. ${ }^{12}$

Now consider the regressive property and the principal/agents setting. Suppose again that all the sets $S_{i}$ are finite. Suppose that for the function $\rho_{1}$, and for some

\footnotetext{
11 For the case where the $S_{i}$ are not finite but are compact, while reward, revenue, and cost functions are smooth, a different argument leads to the same conclusion.

12 In a recent contribution, Nandeibam (2002) presents a related strong result for budget-balancing (but not necessarily nondecreasing) reward functions when the revenue function is smooth and concave and the cost functions are smooth. Let $\bar{x}$ be a strategy profile and let $V$ be a an $n$-player reward profile. It is shown that if there is a game in this class for which $\bar{x}$ is an equilibrium and $V$ is the associated reward profile, then there is also a game which uses constant-share reward functions and again has $\bar{x}$ as an equilibrium and $V$ as the associated reward profile. Hence given a game where some reward functions are sometimes decreasing and there is an equilibrium with a given surplus, we can replace the game by a constant-share game in which the same surplus is obtained at some equilibrium. Constant-share reward functions are nondecreasing.
} 
$x_{-1}^{*} \in S_{-1}$, and some $x_{1}, x_{1}^{\prime} \in S_{-1}$ with $c_{1}\left(x_{1}^{\prime}\right)>c_{1}\left(x_{1}\right)$, we have $A\left(x_{1}^{\prime}, x_{-1}^{*}\right)=$ $A\left(x_{1}, x_{-1}^{*}\right)+\Delta$, with $\Delta>0$, but $\rho_{1}\left(A\left(x_{1}^{\prime}, x_{-1}\right)\right)-\rho_{1}\left(A\left(x_{1}, x_{-1}\right)\right)>\Delta$, so that regressivity is violated. Now replace the reward function $\rho_{1}$ by a new function $\tilde{\rho}_{1}$ which coincides with $\rho_{1}$ at all revenues except $A\left(x_{1}^{\prime}, x_{-1}\right)$. There we have $\tilde{\rho}_{1}\left(A\left(x_{1}^{\prime}, x_{-1}\right)=\right.$ $\rho_{1}\left(A\left(x_{1}, x_{-1}\right)\right)+\Delta$. This change does not eliminate any equilibria of the original game, except possibly $\left(x_{1}^{\prime}, x_{-1}^{*}\right)$. Analagous changes can be made wherever regressivity is violated, until regressivity holds. Then the only equilibria that might be eliminated are profiles which occur after some player increases his effort, where that effort increase leads to a reward jump that exceeds the revenue increase which the extra effort produces. It appears difficult to construct a plausible game in which eliminating such equilibria diminishes the principal's maximal equilibrium residual.

Moreover, in the principal/agents setting, nondecreasing reward functions have the NDR property if the principal insists on reserving a certain proportion of revenue for himself, say the proportion $F$, with $0<F<1$, and lets the agents divide up the remaining portion as they wish, using any reward functions for which $\sum_{i \in N} \rho_{i}(z)=$ $(1-F) \cdot z \cdot{ }^{13}$

Finally, consider the regressivity property and the partnership setting. Every rewardfunction profile is budget-balancing. Hence it is NDR and therefore each indidividual reward function is regressive if all of them are nondecreasing.

\subsection{A summary of some previous results}

The following are some of the conclusions obtained in Courtney and Marschak (2006). Only nondecreasing reward functions are considered. One of the results deals with existence of equilibrium and the others concern the possibility of squandering.

(i) Existence of a (pure-strategy) equilibrium. Suppose the reward functions of a sharing game are linearly related, i.e. there is a function $\rho: A(S) \rightarrow \mathbb{R}$, and positive numbers $r_{1}, \ldots, r_{n}$ such that for each $i$ we have $\rho_{i}=r_{i} \cdot \rho$. Then a pure-strategy equilibrium exists if $S$ contains a maximizer of the function $p: S \rightarrow \mathbb{R}$ defined by

$$
p(x)=\rho(A(x))-\sum_{i \in N} \frac{1}{r_{i}} \cdot c_{i}(x) .
$$

That maximizer is an equilibrium. In particular, every finite game with linearly related rewards has a maximizer of $p$ and hence an equilibrium. One can easily construct games in which the rewards are not linearly related and there is no (pure strategy) equilibrium. ${ }^{14}$ The function $p$ is a potential function for the game, in the sense developed by Monderer and Shapley (1996).

\footnotetext{
13 That is one way to model a principal who licenses a technology to a partnership.

14 Existence of an equilibrium can also be shown, using standard supermodularity results, if each individual payoff function $\rho_{i} A\left(\left(x_{i}, x_{-i}\right)\right)-c_{i}\left(x_{i}\right)$ has the "increasing differences" property: the increment in $i$ 's payoff when $x_{i}$ is replaced by $x_{i}+\Delta$ (where $\Delta>0$ ), is not smaller for $x_{-i}$ than for $x_{-i}^{*}$, where $x_{j}^{*} \geq x_{j}$ for all $j \neq i$. Our existence result does not require the increasing differences property.
} 
(ii) No one squanders in productive $N D R$ games that exhibit complementarity. Complementarity is a property of the pair $\left(A,\left\{c_{i}\right\}_{i \in N}\right)$. A pair (and a game) exhibits complementarity if for all $i \in N$ and any $x_{i}, x_{i}^{\prime} \in S_{i}$ satisfying $c_{i}\left(x_{i}^{\prime}\right) \geq c_{i}\left(x_{i}\right)$, we have w $^{15}$

$$
A\left(y / x_{i}^{\prime}\right)-A\left(y / x_{i}\right) \geq A\left(z / x_{i}^{\prime}\right)-A\left(z / x_{i}\right)
$$

whenever $c_{j}\left(y_{j}\right) \geq c_{j}\left(z_{j}\right)$ for all $j \neq i .{ }^{16}$ If $x$ is an inefficient equilibrium of a sharing game with complementarity and nondecreasing NDR reward functions, and $y$ is efficient, then $c_{i}\left(x_{i}\right) \leq c_{i}\left(y_{i}\right)$ for all $i \in N$. So at the equilibrium $x$, no player squanders relative to the efficient $y$; every player either shirks or spends the same as at $y$. This proposition is not implied by standard theorems on supermodular games and complementarity. It requires its own argument. ${ }^{17}$

(iii) In NDR games which lack complementarity but exhibit "aggregate" productivity there is some set of players who shirk (aggregatively). We shall say that a sharing game exhibits aggregate productivity if for any $s, s^{\prime} \in S$ we have $A\left(s^{\prime}\right) \geq A(s)$ whenever $\tau\left(s^{\prime}\right)>\tau(s)$, and if for any partitioning of the player set $N$ into two nonempty subsets $V$ and $W$ we have $A\left(s_{V}^{\prime}, s_{W}\right) \geq A(s)$ whenever $\tau\left(s_{V}^{\prime}\right)>\tau\left(s_{V}\right)$. (Here $\tau\left(s_{V}\right)$ denotes $\sum_{j \in V} c_{j}\left(s_{j}\right)$, and $\left(s_{V}^{\prime}, s_{W}\right)$ denotes the $n$-tuple whose $j$ th entry is $s_{j}^{\prime}$ or $s_{j}$ according to whether $j \in V$ or $j \in W$ ). Aggregate productivity does not imply productivity and is not implied by productivity. If $y$ is an efficient profile in an aggregatively productive NDR game, and $x$ is an inefficient equilibrium, then in any partitioning of the player set, at least one set of the partitioning aggregatively shirks, i.e., the total spent by the players in that set is less at $x$ than at $y$. Formally, for any partitioning of $N$ into $T \geq 1$ disjoint nonempty subsets $W_{1}, \ldots, W_{T}$, there is a subset $W_{t^{*}}$ for which $\tau\left(x_{W_{t^{*}}}\right)<\tau\left(y_{W_{t^{*}}}\right)$.

(iv) In NDR games with equal rewards there cannot be aggregative squandering at a potential-maximizing equilibrium. Consider an NDR game in which all players have the same nondecreasing reward function. Suppose that $x$ is an inefficient equilibrium and that it maximizes the potential function $p$ which appears in our existence result.

\footnotetext{
15 A symbol of the form $x / \bar{x}_{i}$ denotes the $n$-tuple obtained from $x=\left(x_{1}, \ldots, x_{n}\right)$ when we replace $x_{i}$ by $\bar{x}_{i}$.

16 Consider the case where the game is not an effort game. For some $i, x_{i}, x_{i}^{\prime}$ we have $x_{i}^{\prime} \neq x_{i}$ but $c_{i}\left(x_{i}^{\prime}\right)=c_{i}\left(x_{i}\right)$. Player $i$ 's deviation from $x_{i}$ to $x_{i}^{\prime}$ may increase revenue, decrease it, or leave it unchanged. Complementarity tells us that the change in revenue is not less when the other players choose $y_{-i}$ than when they choose $z_{-i}$.

17 Standard theorems are of some help in the special case of identical constant-share reward functions. One can then use, for example, Theorem 4.2.2 in Topkis (1998), which concerns a collection of supermodular games, each defined by a parameter, and provides conditions under which there exists a greatest and least equilibrium each of which is increasing in the parameter. Let player $i$ 's payoff in a sharing game be $k \cdot A(x)-c_{i}\left(x_{i}\right), 0<k \leq 1$. Our proposition compares an equilibrium profile of the game with an efficient (surplus-maximizing) profile. A necessary condition for a profile to be efficient is that it have the equilibrium property for a game in which $k$ equals one. But to make that a sufficient condition as well, we have to make further assumptions on $A$ and the $c_{i}$. If the $k=1$ equilibrium condition were indeed both necessary and sufficient for efficiency, then one could apply Topkis' Theorem 4.2.2, letting his parameter be our $k$, to obtain some conclusions about what happens to the equilibrium value of the effort $x_{i}$ when we start with $k$ equal to one and then decrease its value. Even so, in the case of multiple equilibria, those conclusions would only cover the least and the greatest, not all of them, as our proposition does. Theorem 4.2.2 is not helpful when we turn from the special case of constant-share functions to the general NDR case.
} 
Then at $x$ there cannot be aggregative squandering relative to an efficient $y$, i.e., we must have $\tau(x) \leq \tau(y)$. That result, combined with our existence result, has an interesting Corollary: If the game has a maximizer of $p$ (if it is a finite game, for example), and if it has just one equilibrium, then that equilibrium must be the unique maximizer of $p$, and it cannot be aggregatively squandering relative to an efficient profile, i.e., it is either efficient or else aggregatively shirking relative to some efficient profile.

Note that the NDR property is assumed in all our propositions about squandering. If we drop that requirement, then squandering can occur even though the other conditions in our propositions are fulfilled. This is illustrated rather strikingly, in the following two-player symmetric example, where squandering dominates for each player.

Let $S_{1}=S_{2}=\{1,2,3\}$. Let the revenue function $A$ be given by the following symmetric smatrix

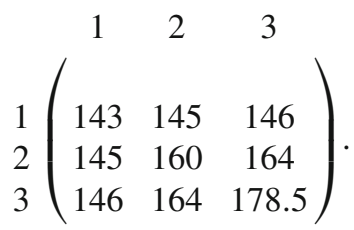

Let $c_{i}\left(x_{i}\right)=9 x_{i}-8, i=1,2$. So a player's possible costs are 1, 10, and 19. We have strict productivity. Note that the "marginal products" (the revenue gains) diminish as a player increases his cost, and the marginal product due to a given cost increment grows when the other player increases her cost. Thus we have complementarity. Surplus is given by:

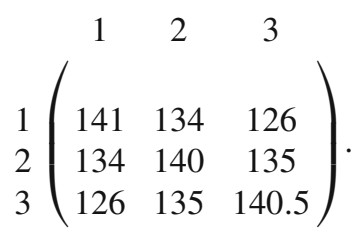

The unique efficient profile is $(1,1)$. Now let each player have the following increasing reward function:

\begin{tabular}{c|c|c|c|c|c|c|} 
revenue: & 143 & 145 & 146 & 160 & 164 & 178.5 \\
\cline { 2 - 7 } reward: & 2 & 14.5 & 23 & 80 & 80.5 & 81
\end{tabular}

This function is not regressive (For example, when revenue rises by 2, from 143 to 145 , reward goes up by 12.5 ). Hence the sharing game lacks the NDR property. The payoff matrix of the game is

\begin{tabular}{|c|c|c|c|}
\hline & 1 & 2 & 3 \\
\hline 1 & $(1,1)$ & $(13.5,4.5)$ & $(22,4)$ \\
\hline 2 & $(4.5,13.5)$ & $(78,78)$ & $(70.5,61.5)$ \\
\hline 3 & $(4,22)$ & $(61.5,70.5)$ & $(62,62)$ \\
\hline
\end{tabular}


For each player the strategy 2 (which costs 10) dominates. At that strategy each player squanders relative to the efficient pair $(1,1)$, where each player's cost is only 1 .

\section{Technological change and squandering equilibria}

A strong motivation for understanding squandering and shirking arises when one asks whether improved technology might change the organization's structure because the improvement makes sharing games more attractive. Suppose the cost functions drop. One would want to know whether such an improvement lessens the "decentralization penalty" associated with an equilibrium of the sharing game, i.e., the amount by which surplus at the equilibrium falls short of its maximum. If an organization contemplates the use of a sharing game because of its informational simplicity and the autonomy it permits, rather than using (for example) the costly and intrusive monitoring that would guarantee maximal surplus, then a drop in the penalty associated with a sharing-game equilibrium may tip the balance in favor of the sharing game. It turns out, however, that the effect of a cost drop on the penalty associated with an equilibrium is generally quite different when the equilibrium is squandering than when it is shirking.

Consider a sharing game where each player $i$ 's cost function is $c_{i}=\gamma \cdot \bar{c}_{i}$, and $\gamma>0$ is a parameter which drops when technology improves, because the inputs which players must buy (to carry out a chosen strategy) become cheaper or the input quantities needed are smaller. Suppose first that the drop in $\gamma$ is small enough so that every strategy profile which was efficient before the drop remains efficient and every profile which was a sharing-game equilibrium remains an equilibrium. Let $\bar{\tau}(x)$ denote $\sum_{i \in N} \bar{c}_{i}(x)$. Then if $x^{*}$ is efficient while $\tilde{x}$ is an equilibrium, the decentralization penalty (surplus shortfall) associated with $\tilde{x}$ is

$$
\left[A\left(x^{*}\right)-\gamma \cdot \bar{\tau}\left(x^{*}\right)\right]-[A(\tilde{x})-\gamma \cdot \bar{\tau}(\tilde{x})]=A\left(x^{*}\right)-A(\tilde{x})+\gamma \cdot\left[\bar{\tau}(\tilde{x})-\bar{\tau}\left(x^{*}\right)\right] .
$$

If $\tilde{x}$ is squandering relative to $x^{*}$, then the final expression in square brackets is positive and so the penalty drops as $\gamma$ drops. But if $\tilde{x}$ is shirking relative to $x^{*}$, then that expression is negative and the penalty rises as $\gamma$ drops.

Suppose, on the other hand, that each strategy set $S_{i}$ is a subset of the real line, and that for every $\gamma$ we can select an efficient profile and an equilibrium in the sharinggame defined by $\gamma$, in such a way that both of them change smoothly as $\gamma$ changes. If the selected equilibrium is always squandering relative to the selected efficient profile, if the game has regressive reward functions (which is the case for NDR games with nondecreasing rewards), and if we make the plausible assumption ${ }^{18}$ that each player's equilibrium effort does not drop when $\gamma$ drops, then we can again make the unambiguous claim that technological improvement (i.e., a drop in $\gamma$ ) lowers the penalty associated with the squandering equilibrium. We have the theorem which follows. Each cost function again has the form $c_{i}\left(x_{i}\right)=\gamma \bar{c}_{i}\left(x_{i}\right)$ and we again let $\bar{\tau}(x)$ denote $\sum_{i \in N} \bar{c}_{i}\left(x_{i}\right)$. In stating the theorem, we let $\pi\left(\tilde{x}, x^{*}\right)$ denote the decentralization

18 Assumption (vi) in the theorem which follows. 
penalty associated with the equilibrium $\tilde{x}$ and the efficient profile $x^{*}$, i.e., $\pi\left(\tilde{x}, x^{*}\right)=$ $A\left(x^{*}\right)-\tau\left(x^{*}\right)-[A(\tilde{x})-\tau(\tilde{x})]$.

Theorem A Consider a sharing game $\left\langle\left\{S_{i}\right\}_{i \in N}, A,\left\{\rho_{i}\right\}_{i \in N},\left\{\gamma \bar{c}_{i}\right\}_{i \in N}\right\rangle$ in which the following hold:

(i) Each strategy set $S_{i}$ is an open set in the real line.

(ii) for all $\gamma \in\left[\gamma^{+}, \gamma^{++}\right]$, where $0<\gamma^{+}<\gamma^{++}$, there is an efficient profile $x^{*}(\gamma)=\left(x_{1}^{*}(\gamma), \ldots, x_{n}^{*}(\gamma)\right)$ and an equilibrium $\tilde{x}(\gamma)=\left(\tilde{x}_{i}(\gamma), \ldots, \tilde{x}_{n}(\gamma)\right)$, where the functions $x_{i}^{*}, \tilde{x}_{i}$ are differentiable on $\left[\gamma^{+}, \gamma^{++}\right]$

(iii) $A$ is differentiable, with respect to each of its arguments, on $S=S_{1} \times \cdots \times S_{n}$

(iv) each function $\bar{c}_{i}$ is differentiable on $S_{i}$ and is strictly increasing

(v) each reward function $\rho_{i}$ is positive-valued, differentiable on $A(S)$, regressive, and nondecreasing.

\section{Suppose that}

(vi) for all $i \in N$ we have $\tilde{x}_{i}\left(\gamma^{\prime}\right) \geq \tilde{x}_{i}\left(\gamma^{\prime \prime}\right)$ whenever $\gamma^{+} \leq \gamma^{\prime}<\gamma^{\prime \prime} \leq \gamma^{++}$.

Suppose that for $\gamma^{+} \leq \gamma^{\prime}<\gamma^{\prime \prime} \leq \gamma^{++}$, the equilibrium $\tilde{x}\left(\gamma^{\prime}\right)$ is aggregatively squandering relative to $x^{*}\left(\gamma^{\prime}\right)$ and $\tilde{x}\left(\gamma^{\prime \prime}\right)$ is aggregatively squandering relative to $x^{*}\left(\gamma^{\prime \prime}\right)$, i.e., $\gamma^{\prime} \bar{\tau}\left(\tilde{x}\left(\gamma^{\prime}\right)\right)>\gamma^{\prime} \bar{\tau}\left(x^{*}\left(\gamma^{\prime}\right)\right), \gamma^{\prime \prime} \bar{\tau}\left(\tilde{x}\left(\gamma^{\prime \prime}\right)\right)>\gamma^{\prime \prime} \bar{\tau}\left(x^{*}\left(\gamma^{\prime \prime}\right)\right)$. Then $\pi\left(\tilde{x}\left(\gamma^{\prime \prime}\right)\right.$, $\left.x^{*}\left(\gamma^{\prime \prime}\right)\right)<\pi\left(\tilde{x}\left(\gamma^{\prime}\right), x^{*}\left(\gamma^{\prime}\right)\right)$ (the penalty associated with the squandering equilibrium drops when technology improves).

Proof Define $V(\gamma)$ to be the penalty associated with $\tilde{x}(\gamma), x^{*}(\gamma)$, i.e., $V(\gamma)=$ $\pi\left(\left(\tilde{x}(\gamma), x^{*}(\gamma)\right)\right.$. We shall consider the derivative $V^{\prime}$ and shall prove that it is positive for $\gamma \in\left[\gamma^{+}, \gamma^{++}\right]$, which will imply our claim. We can write $V(\gamma)=V_{a}(\gamma)-V_{b}(\gamma)$, where

$$
V_{a}(\gamma)=A\left(x^{*}(\gamma)\right)-\gamma \cdot \bar{\tau}\left(x^{*}(\gamma)\right), \quad V_{b}(\gamma)=A(\tilde{x}(\gamma))-\gamma \cdot \bar{\tau}(\tilde{x}(\gamma)) .
$$

Since $x^{*}(\gamma)$ is a maximizer of $A(x)-\gamma \bar{\tau}(x)$, it follows from the Envelope Theorem that

$$
V_{a}^{\prime}(\gamma)=-\bar{\tau}\left(x^{*}(\gamma)\right)
$$

Whenever $x$ is an equilibrium for the game defined by a fixed $\gamma$, the first-order condition $\rho_{i}^{\prime}(A(x)) \cdot A_{i}(x)=\gamma \cdot \bar{c}_{i}^{\prime}\left(x_{i}\right)$ is met for each $i$, where $A_{i}$ denotes the partial derivative of $A$ with respect to $x_{i}$. In particular, for the equilibrium $\tilde{x}(\gamma)$, the condition can be rewritten as

$$
A_{i}(\tilde{x}(\gamma))=\frac{\gamma \bar{c}_{i}^{\prime}\left(\tilde{x}_{i}(\gamma)\right)}{\rho_{i}^{\prime}(A(\tilde{x}(\gamma))}
$$


Differentiating $V_{b}$, and using (++), we obtain

$$
\begin{aligned}
V_{b}^{\prime}(\gamma) & =\sum_{i \in N}\left[A_{i}(\tilde{x}(\gamma)) \cdot \tilde{x}_{i}^{\prime}(\gamma)-\gamma \bar{c}_{i}^{\prime}\left(\tilde{x}_{i}(\gamma)\right) \cdot \tilde{x}_{i}^{\prime}(\gamma)-\bar{c}_{i}\left(\tilde{x}_{i}(\gamma)\right)\right] \\
& =\sum_{i \in N}\left[\frac{\gamma \bar{c}_{i}^{\prime}\left(\tilde{x}_{i}(\gamma)\right)}{\rho_{i}^{\prime}(A(\tilde{x}(\gamma))} \cdot \tilde{x}_{i}^{\prime}(\gamma)-\gamma \bar{c}_{i}^{\prime}\left(\tilde{x}_{i}(\gamma)\right) \cdot \tilde{x}_{i}^{\prime}(\gamma)\right]-\bar{\tau}(\tilde{x}(\gamma)) \\
& =\sum_{i \in N}\left[\gamma \bar{c}_{i}^{\prime}\left(\tilde{x}_{i}(\gamma)\right) \cdot \tilde{x}_{i}^{\prime}(\gamma) \cdot\left(\frac{1}{\rho_{i}^{\prime}(A(\tilde{x}(\gamma))}-1\right)\right]-\bar{\tau}(\tilde{x}(\gamma))
\end{aligned}
$$

We therefore obtain, using (+),

$$
\begin{aligned}
V^{\prime}(\gamma) & =V_{a}^{\prime}(\gamma)-V_{b}^{\prime}(\gamma) \\
& =\bar{\tau}(\tilde{x}(\gamma))-\bar{\tau}\left(x^{*}(\gamma)\right)-\sum_{i \in N}\left[\gamma \bar{c}_{i}^{\prime}\left(\tilde{x}_{i}(\gamma)\right) \cdot \tilde{x}_{i}^{\prime}(\gamma) \cdot\left(\frac{1}{\rho_{i}^{\prime}(A(\tilde{x}(\gamma))}-1\right)\right] .
\end{aligned}
$$

Since $\rho_{i}$ is regressive, we have (as noted just after the definition of regressivity) that $\rho_{i}^{\prime} \leq 1$. Hence $\frac{1}{\rho_{i}^{\prime}}-1 \geq 0$. Since, by assumption, $\bar{c}_{i}^{\prime} \geq 0$ and $\tilde{x}_{i}^{\prime} \leq 0$, the entire expression in large square brackets is nonpositive. By our squandering assumption, $\bar{\tau}(\tilde{x}(\gamma))>\bar{\tau}\left(x^{*}(\gamma)\right)$. We conclude that $V^{\prime}(\gamma)>0$ for $\gamma \in\left[\gamma^{+}, \gamma^{++}\right]$.

If $\tilde{x}(\gamma)$ is a shirking equilibrium, the situation changes. Unless we make further assumptions, the sign of $V^{\prime}$-i.e., the effect of technological improvement on the penalty associated with the equilibrium - is ambiguous.

Theorem A concerns games with regressive reward functions in which the strategy sets are continua and there are squandering equilibria. We have not yet illustrated a game of that sort. (Finite games with squandering are illustrated in the next section). We now provide an "economic" example of such a game and we apply Theorem A to the example.

There are two players. They produce the same product and each uses his own plant to do so. The plants are identical. Each player chooses his product quantity and pays its cost, but they share the revenue obtained when the sum of the two quantities is sold. Player $i$ chooses a nonnegative quantity $x_{i}$, and that costs $\gamma \ln \left(1+x_{i}\right)$ (so, in the notation of Theorem A, $\left.\bar{c}_{i}\left(x_{i}\right)=\ln \left(1+x_{i}\right)\right)$. When the total $x_{1}+x_{2}$ is placed on the market, it sells for a price per unit of $\alpha-\left(x_{1}+x_{2}\right)$, where $\alpha>0$. For simplicity, we permit negative prices; they will not occur at efficient profiles or at equilibria. Revenue at $\left(x_{1}, x_{2}\right)$ is $A\left(x_{1}, x_{2}\right)=\left(\alpha-x_{1}-x_{2}\right)\left(x_{1}+x_{2}\right)$. (This function $A$ violates productivity when the sum of the two strategies exceeds $\frac{\alpha}{2}$, but Theorem A does not assume productivity). Since, in each plant, marginal cost always drops when product grows, efficiency is achieved if just one plant is used-say Player 1's plant. Then the profile $(y, 0)$, with $y>0$, is efficient if $y$ maximizes $\pi(y)=(\alpha-y)(y)-\gamma \ln (1+y)$ on the nonnegative reals. The function $\pi$ is defined for all $y>-1$. We have $\pi^{\prime}(y)=\alpha-2 y-\frac{\gamma}{1+y}$. Note the following four facts: (i) $\pi(0)=0$; (ii) $\pi^{\prime}(0)=\alpha-\gamma$; (iii) $\lim _{y \rightarrow-1} \pi(y)=\infty$; (iv) $\lim _{y \rightarrow \infty} \pi(y)=-\infty$. The first-order condition for a maximum is $0=\pi^{\prime}(y)$, which is equivalent to $0=2 y^{2}-w y+(\gamma-w-2)$, where $w=\alpha-2$. The roots of 
that equation are $\frac{1}{4}\left(w \pm \sqrt{w^{2}-8 \gamma+8 w+16}\right)$. If $\alpha>\gamma$, then these are both real, so the graph of $\pi$ reverses direction twice. Assume that $\alpha>\gamma$ and call the larger of the two roots $y^{*}(\gamma)$. In view of (i)-(iv), the graph of $\pi$ has an arbitrarily high value when $y$ is close to -1 ; descends to a negative number when $y$ equals the smaller of the two roots (that root is negative); rises to zero at $y=0$; continues to rise until $y$ equals $y^{*}(\gamma)$; and then decreases without bound. (In informal economic terminology: at both roots marginal revenue equals marginal cost and both marginals are falling; at $y^{*}(\gamma)$ marginal revenue is falling faster than marginal cost, but at the smaller of the two roots the opposite is true). Thus the profile $x^{*}(\gamma)=\left(y^{*}(\gamma), 0\right)$ is efficient if $\alpha>\gamma$.

Now consider the sharing game in which each player chooses a positive quantity and gets $r$ times revenue, where $0<r \leq \frac{1}{2}$. Suppose we have, for some $\gamma$, an equilibrium $\tilde{x}(\gamma)$ of the form $(z(\gamma), z(\gamma))$. That equilibrium is squandering relative to $x^{*}(\gamma)$ if $2 \gamma \cdot \ln (1+\gamma)>\gamma \cdot \ln \left(1+y^{*}(\gamma)\right.$, which is equivalent to $(1+z(\gamma))^{2}>1+y^{*}(\gamma)$. We can, in fact, find $\alpha, \gamma^{+}, \gamma^{++}$, with $0<\gamma^{+}<\gamma^{++}<\alpha$ such that for $r=\frac{1}{2}$ and for every $\gamma \in\left[\gamma^{+}, \gamma^{++}\right]$, such a squandering equilibrium $(z(\gamma), z(\gamma))$ exists. Moreover, the condition $\tilde{x}_{i}^{\prime}(\gamma)<0, i=1,2$, required in Assumption (vi) of Theorem A, is satisfied for every $\gamma \in\left[\gamma^{+}, \gamma^{++}\right]$. Then Theorem A implies that if the sharing game uses $r=\frac{1}{2}$, then for $\gamma^{+}<\gamma^{\prime}<\gamma^{\prime \prime}<\gamma^{++}$, the decentralization penalty associated with $\tilde{x}\left(\gamma^{\prime}\right)$ is less than the penalty associated with $\tilde{x}\left(\gamma^{\prime \prime}\right) .{ }^{19}$

19 The argument for the existence of such a triple $\left(\alpha, \gamma^{+}, \gamma^{++}\right)$is as follows. Suppose, to start with, that Player $i$ 's strategy set is the closed set $\left\{x_{i}: x_{i} \geq 0\right\}$. Given a value of $x_{2}$, Player 1's net payoff for $x_{1}$ is $P\left(x_{1} ; x_{2}\right)=r \cdot\left(\alpha-x_{1}-x_{2}\right) \cdot\left(x_{1}+x_{2}\right)-\gamma \ln \left(x_{1}+1\right)$. That function is defined for $x_{1}>-1$. Similarly to what we found above for $\pi$, we have

$$
\lim _{x_{1} \rightarrow-1} P\left(x_{1} ; x_{2}\right)=\infty, \quad \lim _{x_{1} \rightarrow \infty} P\left(x_{1} ; x_{2}\right)=-\infty
$$

We have $P^{\prime}\left(x_{1} ; x_{2}\right)=r \alpha-2 r x_{1}-2 r x_{2}-\frac{\gamma}{x_{1}+1}$. The first-order equation $P^{\prime}\left(x_{1}, x_{2}\right)=0$ is equivalent to $0=2 x_{1}^{2}+x_{1} \cdot\left(-\alpha+2+2 x_{2}\right)+\left(\gamma / r+2 x_{2}-\alpha\right)$. This has the two roots $\frac{1}{4} \cdot\left(w-2 x_{2} \pm\right.$ $\left.\sqrt{\left(2 x_{2}-w\right)^{2}-8\left(\gamma / r+2 x_{2}-w-2\right)}\right)$, where $w$ again denotes $\alpha-2$. Suppose now that the roots are real and call the larger of the roots $L\left(x_{2}\right)$. Then, in view of $(+)$, the graph of $P\left(x_{1} ; x_{2}\right)$ is similar to the graph of $\pi$ : it reverses direction twice, and the value of $P$ at $L\left(x_{2}\right)$ exceeds its value at the smaller root. One or both roots may, however, be negative. Player 1's best response to Player 2's $x_{2}$ is:

$$
\hat{x}_{1}\left(x_{2}\right)=\left\{\begin{array}{ll}
L\left(x_{2}\right) & \text { if } L\left(x_{2}\right)>0 \\
0 & \text { otherwise }
\end{array} \text { and } P\left(L\left(x_{2}\right) ; x_{2}\right)>P\left(0 ; x_{2}\right)\right.
$$

Player 2's best response to $x_{1}$, denoted $\hat{x}_{2}\left(x_{1}\right)$, is analagous. Now fix $\alpha$ and $r$. Suppose we find a positive value of $\gamma$ and a positive quantity $z(\gamma)$ such that $z(\gamma)=\hat{x}_{1}(z(\gamma))=L(z(\gamma))$, which, by symmetry, implies $z(\gamma)=\hat{x}_{2}(z(\gamma))$. Then the profile $\tilde{x}(\gamma)=(z(\gamma), z(\gamma))$ is an equilibrium of the game and $z(\gamma)$ is the larger (real) root of the equation $P^{\prime}(z ; z)=0$. Thus $z(\gamma)$ is a solution of the equation $(3 / 2) z-(1 / 4) w=$ $(1 / 4) \cdot \sqrt{(2 z-w)^{2}-8(\gamma / r+2 z-w-2)}$. Multiplying both sides by 4 and then squaring both sides, we obtain $36 z^{2}-12 w z+w^{2}=4 z^{2}-4 w z+w^{2}-8 \frac{\gamma}{r}+16 z-8 w+16$.

Collecting terms and simplifying, we get $4 z^{2}-(w-2) z+\left(\frac{\gamma}{r}-w-2\right)=0$. Now choose $z(\gamma)$ to be the larger of the two solutions of that equation, i.e., $z(\gamma)=\frac{1}{8} \cdot\left(w-2+\sqrt{\left.(w-2)^{2}-16 \frac{\gamma}{r}-w-2\right)}\right)$. To summarize: given positive $r, \gamma, \alpha$, we have a squandering equilibrium $(z(\gamma), z(\gamma))$, where $z(\gamma)=$ $\frac{1}{8} \cdot\left(w-2+\sqrt{\left.(w-2)^{2}-16 \frac{\gamma}{r}-w-2\right)}\right)$ and $w=\alpha-2$, if: (I) $\alpha>\gamma$; (II) $z(\gamma)$ is real; (III) $z(\gamma)>0$; (IV) $P(z(\gamma) ; z(\gamma)) \geq P(0 ; z(\gamma))$, which is equivalent to $\alpha>3 z(\gamma)$; (V) $(1+z(\gamma))^{2}>1+y^{*}(\gamma)$. Now 


\section{Squandering and the Substitutes property}

As we saw in 2.3, complementarity rules out squandering by any player at any equilibrium of any productive NDR game, whatever the (increasing) cost functions may be. We have seen that squandering equilibria are important. It is natural to see whether the "opposite" of complementarity, namely the Substitutes property, implies the existence of such equilibria. A productive sharing game has that property if the marginal product (the revenue gain) achieved when one player increases his effort is damaged (or at least not enhanced) if at least one other player increases his effort. Whether a sufficiently strong Substitutes property implies squandering turns out to be a challenging question. We offer small steps towards an answer.

In Courtney and Marschak (2006), we proved a theorem ${ }^{20}$ about a class of symmetric two-person games with linear costs and constant-share rewards. Player $i$ 's strategy set is a closed interval $T_{i}=\left[g_{i}, h_{i}\right] \subset \mathbb{R}^{+}$. The revenue function $A$ is twice differentiable on $T_{1} \times T_{2}$. At every strategy pair $\left(x_{1}, x_{2}\right)$, we have the Substitutes property $\frac{\partial^{2} A}{\partial x_{1} \partial x_{2}} \leq 0$, as well as the Diminishing Marginal Product (DMP) property $\frac{\partial^{2} A}{\partial x_{i} \partial x_{i}}<0, i=1,2$. A strategy pair $\alpha=(g+v, g+w)$ in the interior of $T_{1} \times T_{2}$ is considered, where $2 g+v+w>\max \left[g_{1}+h_{2}, g_{2}+h_{1}\right]$, which means that $\alpha$ costs more than the corner pairs $\left(g_{1}, h_{2}\right)$ and $\left(h_{1}, g_{2}\right)$. It is shown that if $\alpha$ is an equilibrium of the sharing game, and if, at every strategy pair $\left(x_{1}, x_{2}\right) \in T_{1} \times T_{2}$, the strength of the Substitutes effect (measured by $\left|\frac{\partial^{2} A}{\partial x_{1} \partial x_{2}}\right|$ ) is sufficiently large relative to the strength of the DMP effects (measured by $\left|\frac{\partial^{2} A}{\partial x_{i} \partial x_{i}}\right|, i=1,2$ ), then the corner pairs $\left(g_{1}, h_{2}\right),\left(h_{1}, g_{2}\right)$ are efficient, so that $\alpha$ is squandering.

We now seek analogous propositions for finite games.

Consider a finite two-player game, where each player has $t$ strategies, labelled $1, \ldots, t$. Strategy $\ell+1$ costs more than strategy $\ell$ and the game is strictly productive, so that revenue rises when one player moves to a higher strategy. Let $A_{i j}$ denote revenue at the pair $(i, j)$, where $i$ is 1 's strategy and $j$ is 2's strategy. The game has the DMP property if for every $j \in\{1, \ldots, t\}$, and for $i \in\{2, \ldots, t-1\} 1$ 's DMP effect at $(i, j)$, defined as

\section{Footnote 19 continued}

consider the following case: $\alpha=62$ (so $w=60$ ); $r=\frac{1}{2}$. We first consider $\gamma=1$ and then $\gamma=2$ and $\gamma \in] 1,2\left[\right.$. We have $y^{*}(1)=\frac{1}{4}\left(60+\sqrt{3600-8+480+16}=\frac{1}{4}(60+\sqrt{4083}) \approx \frac{1}{4}(60+63.94) \approx 31\right.$, and $z(1)=\frac{1}{8}(58+\sqrt{3364-32-60-2})=\frac{1}{8}(58+\sqrt{3270}) \approx \frac{1}{8} \cdot(58+57.18) \approx 14.4$. The squandering condition $(\mathrm{V})$ is satisfied, since $(12.89)^{2}>27$. The other four conditions are satisfied as well. Now let $\gamma=2$. We obtain $y^{*}(2)=\frac{1}{4}(50+\sqrt{2900}) \approx 25.963$ and $z(2)=\frac{1}{8}(48+\sqrt{2190}) \approx 11.85$. The squandering condition (V) again holds, and so do the other conditions. The same is true for every $\gamma$ in ]1, 2[.

As claimed, the condition $\tilde{x}_{i}^{\prime}(\gamma)<0, i=1,2$, required in Assumption (vi) of Theorem A, is satisfied for $\gamma \in[1,2]$. Since for all $\gamma \in[1,2], y^{*}(\gamma), z(\gamma)$ are interior points of the closed strategy set $\left\{x_{i}: x_{i} \geq 0\right\}$, we can replace that closed strategy set with the open set $\left\{x_{i}: x_{i}>0\right\}$, as Theorem A requires. Then Theorem A implies that if the sharing game uses $r=\frac{1}{2}$, then for $\alpha=62$, and $\gamma^{+}=1<\gamma^{\prime}<\gamma^{\prime \prime}<2=\gamma^{++}$, the decentralization penalty associated with $\tilde{x}\left(\gamma^{\prime}\right)$ is less than the penalty associated with $\tilde{x}\left(\gamma^{\prime \prime}\right)$. (That can also be checked directly).

20 Theorem 8. 


$$
\left[A_{i j}-A_{i-1, j}\right]-\left[A_{i+1, j}-A_{i, j}\right],
$$

is positive, and every DMP effect for Player 2 (defined analagously) is positive.

The game has the Substitutes property if for $i \in\{2, \ldots, t\}$ and $j$ in $\{1, \ldots, t-1\}$, Player 1's Substitutes effect at $(i, j)$, defined as

$$
\left[A_{i j}-A_{i-1, j}\right]-\left[A_{i, j+1}-A_{i-1, j+1}\right],
$$

is positive, and every Substitutes effect for Player 2 (defined analagously) is positive. ${ }^{21}$ Roughly speaking, the DMP effect is the damage to a player's marginal product due to that player working one level harder. The Substitutes effect is the damage to the player's marginal product due to the other player working one level harder.

\subsection{A class of symmetric two-player finite games with the Substitutes and DMP properties but $\mathrm{NO}$ squandering equilibria}

It will be useful to begin with a "benchmark" class, in which each player has a set of $t$ strategies, namely $\{1,2, \ldots, t\}, t \geq 4$. Revenue at the strategy pair $(i, j)$ is denoted $A_{i j}$. We shall call the game a Simple Substitutes Game if (i) we have symmetry, i.e., $A_{i j}=A_{j i}$; (ii) there is a number $J$ and a strictly decreasing sequence of $2 t-2$ nonnegative numbers, say $\epsilon_{1}, \epsilon_{2}, \ldots, \epsilon_{2 t-2}$, such that $A_{11}=J$ and for every other $(i, j) \in\{1, \ldots, t\} \times\{1, \ldots, t\}$, the revenue for the profile $(i, j)$ is

$$
A_{i j}=J+\epsilon_{1}+\epsilon_{2}+\cdots+\epsilon_{i+j-2}
$$

(iii) Player $i$ 's cost for the strategy $x_{i} \in\{1,2, \ldots, t\}$ is $k \cdot x_{i}$, where $k>0$; (iv) each

\begin{tabular}{|c|c|c|c|c|}
\hline & 1 & 2 & 3 & 4 \\
\hline 1 & $J$ & $J+\epsilon_{1}$ & $J+\epsilon_{1}+\epsilon_{2}$ & $J+\epsilon_{1}+\epsilon_{2}+\epsilon_{3}$ \\
\hline 2 & $J+\epsilon_{1}$ & $J+\epsilon_{1}+\epsilon_{2}$ & $J+\epsilon_{1}+\epsilon_{2}+\epsilon_{3}$ & $J+\epsilon_{1}+\epsilon_{2}+\epsilon_{3}+\epsilon_{4}$ \\
\hline 3 & $J+\epsilon_{1}+\epsilon_{2}$ & $J+\epsilon_{1}+\epsilon_{2}+\epsilon_{3}$ & $\begin{array}{c}J+\epsilon_{1}+\epsilon_{2} \\
+\epsilon_{3}+\epsilon_{4}\end{array}$ & $\begin{array}{l}J+\epsilon_{1}+\epsilon_{2} \\
\quad+\epsilon_{3}+\epsilon_{4}+\epsilon_{5}\end{array}$ \\
\hline 4 & $\begin{array}{l}J+\epsilon_{1}+\epsilon_{2} \\
+\epsilon_{3}\end{array}$ & $\begin{array}{c}J+\epsilon_{1}+\epsilon_{2} \\
+\epsilon_{3}+\epsilon_{4}\end{array}$ & $\begin{array}{l}J+\epsilon_{1}+\epsilon_{2}+\epsilon_{3} \\
\quad+\epsilon_{4}+\epsilon_{5}\end{array}$ & $\begin{array}{c}J+\epsilon_{1}+\epsilon_{2}+\epsilon_{3} \\
+\epsilon_{4}+\epsilon_{5}+\epsilon_{6}\end{array}$ \\
\hline
\end{tabular}
player's reward is half of revenue. To illustrate, here is the revenue matrix for $t=4$. Player 1 chooses a row and Player 2 chooses a column.

Note that in a Simple Substitutes Game:

- We have the Diminishing Marginal Product property.

- We have the Substitutes property.

\footnotetext{
$\overline{21}$ If the game is symmetric $\left(A_{i j}=A_{j i}\right)$ then the statements about Player 2 follow from the statements about Player 1 .
} 
- At any pair $(i, j)$ for which the Substitutes and DMP effects are defined, the two effects are equal.

- For each $T$ in $\{2,3, \ldots, 2 t-1\}$ define the $T$ th antidiagonal to be the set of profiles $\{(r, s): r+s=T\}$. In (or "along") each antidiagonal, total revenue is constant and so is total cost (since both players have the same linear cost function).

Theorem B In a Simple Substitutes Game, an inefficient equilibrium cannot be squandering relative to an efficient profile. ${ }^{22}$

Proof See Appendix.

4.2 Symmetric two-player finite games in which a sufficiently strong Substitutes

Penalty implies that there are squandering equilibria

Theorem B tells us that we have to depart from the structure of Simple Substitutes Games if we are to find squandering equilibria.

Consider a symmetric game with $t \geq 4$ strategies for each player. For $(i, j)$ such that $1<i<t, 1 \leq j<t$, the following definition will be useful.

The Substitutes Penalty $(S P)$ at $(i, j)$ is $\left[A_{i+1, j}-A_{i j}\right]-\left[A_{i, j+1}-A_{i-1, j+1}\right]$.

When the penalty ${ }^{23}$ is positive, it has the following interpretation for games which have the DMP property. Consider Player 1's marginal product due to increasing effort from $i$ to $i+1$ when 2 's effort is $j$. If 2 now raises his effort to $j+1$, then the damage to 1's marginal productivity (the Substitutes effect) is so severe that 1's marginal product now drops, even when 1 reduces his effort and starts at $(i-1, j+1)$ (which gives him a higher marginal product then he has at $(1, j+1)$ because of the DMP effect). The penalty measures the difference between 1 's original marginal product and his new lower one, after 1 has dropped his effort by one level while 2 has raised his effort by one level. Note also that the penalty equals

$$
\begin{aligned}
& \text { [the Substitutes effect } \left.\left(A_{i+1, j}-A_{i j}\right)-\left(A_{i+1, j+1}-A_{i, j+1}\right)\right] \\
& -\left[\text { the DMP effect }\left(A_{i, j+1}-A_{i-1, j+1}\right)-\left(A_{i+1, j+1}-A_{i, j+1}\right)\right] .
\end{aligned}
$$

Note that in a Simple Substitutes Game every SP is zero.

We now consider symmetric $t$-strategy sharing games $(t \geq 4)$ for which the cost functions are $c_{i}\left(x_{i}\right)=k x_{i}, k>0$ and each player's reward is half of revenue. ${ }^{24}$ Each game will have the DMP and Substitutes properties. It will, moreover, satisfy four conditions, each of which concerns the strength of the Substitutes effects. Those conditions, denoted $\mathrm{H} 1-\mathrm{H} 4$, are as follows:

\footnotetext{
22 An application of Theorem B is given in Example 2 of Sect. 5 below.

23 It would be more descriptive to call the quantity we have just defined Player 1's Substitutes Penalty at $(i, j)$. Player 2's Substitutes Penalty at $(i, j)$ would have an analagous definition. Because of symmetry it suffices to consider Player 1 and to use the single definition above.

24 It is straightforward to reinterpret the theorem which follows, and its proof, so that it covers the case where each player receives $r$ times the revenue, where $0<r<\frac{1}{2}$ and costs are linear. Simply replace the revenue $A$ with $r A$ and replace the cost $k x_{i}$ with $2 r k x_{i}$.
} 
H1 : For all $i, j$ satisfying $1<i=j<t$, the Substitutes Penalty at $(i, j)$ is at least $k$.

H2 : For all $i, j$ satisfying $1<i<j<t$, the Substitutes Penalty at $(i, j)$ is at least zero.

H3 : For some $i^{\prime}, j^{\prime}$ satisfying $1<i^{\prime}<j^{\prime}<t$, the Substitutes Penalty at $(i, j)$ is at least $k$.

H4: $A_{2 t}-A_{1 t} \leq k$.

Note that $\mathrm{H} 4$ holds if the Substitutes effects associated with the profiles $(1, j)$ are sufficiently strong that Player 1's marginal product at strategy 1(the additional revenue due to replacing strategy 1 with strategy 2) drops to $k$ or less when player 2 reaches $t$, his costliest strategy.

Theorem C Consider a symmetric strictly productive two-player $t$-strategy game with the Substitutes and DMP properties. Suppose $t \geq 4$, and for $i=1,2$, we have $c_{i}\left(x_{i}\right)=k x_{i}$, where $k>0$. Suppose H1-H4 hold. Then

(i) $(1, t)$ and $(t, 1)$ are efficient

(ii) if $(i, j)$, with $i \leq j$, is an equilibrium, then $j \in\{t, t-1,1\}$.

Proof See Appendix. ${ }^{25}$

Corollary to Theorem $C$ Consider a game that meets the conditions of Theorem $\mathrm{C}$. Let $\left(i^{*}, j^{*}\right)$ be an inefficient equilibrium and suppose that $\left(i^{*}, j^{*}\right) \notin\{(1,1),(2, t-1)$, $(t-1,2)\}$. Then $\left(i^{*}, j^{*}\right)$ is squandering relative to every efficient profile.

Proof Claims 1-3 in the proof of Theorem C imply that $(1, t)$ and $(t, 1)$ are efficient and that no other profiles are efficient except possibly $(1,1)$. Suppose $i^{*} \leq j^{*}$. Theorem $\mathrm{C}$ implies that $j^{*} \in\{t, t-1\}$. Since $\left(i^{*}, j^{*}\right) \notin\{(1,1),(2, t-1),(t-1,2)\}$, we conclude that $i^{*}+j^{*}>t+1$. If $i^{*} \geq j^{*}$, a symmetric argument leads to the same conclusion. Thus $\left(i^{*}, j^{*}\right)$ is indeed squandering relative to every efficient profile. ${ }^{26}$

Note, in particular, that any equilibrium other than $(1,1)$ in which both players choose the same effort level is squandering.

For a six-strategy example illustrating Theorem $\mathrm{C}$, let $k=10$ and consider the following revenue table. The column chooser (Player 2) has marginal products-for a fixed row choice by Player1 - that are given as single-box numbers. The row chooser (Player 1) has marginal products - for a fixed column choice by Player 2-that are given as double-box numbers. Thus the DMP effects are the drops in the double-box quantitities as we go down a column of double boxes and the drops in the single-box quantities as we go along a row of single boxes. The Substitutes effects are the drops in the double-box quantitities as we go along a row of double boxes and the drops in the single-box quantities as we go down a column of single boxes. A Substitutes

\footnotetext{
25 We are grateful to Kevin Lin for generalizing a previous proof for the case $t=4$ to the general case.

26 If we replace strict productivity by productivity, then we obtain a version of Theorem $\mathrm{C}$ where, in the conclusion, $\{t, t-1,1\}$ is replaced by $\{t, t-1, t-2,1\}$. Then the (weaker) Corollary says that any inefficient equilibrium which is not $(1,1),(2, t-1)$, or $(3, t-2)$ (or their symmetric counterparts) must be squandering.
} 
Penalty is the drop when we go from a double-box quantity to the double-box quantity that lies directly "northeast".

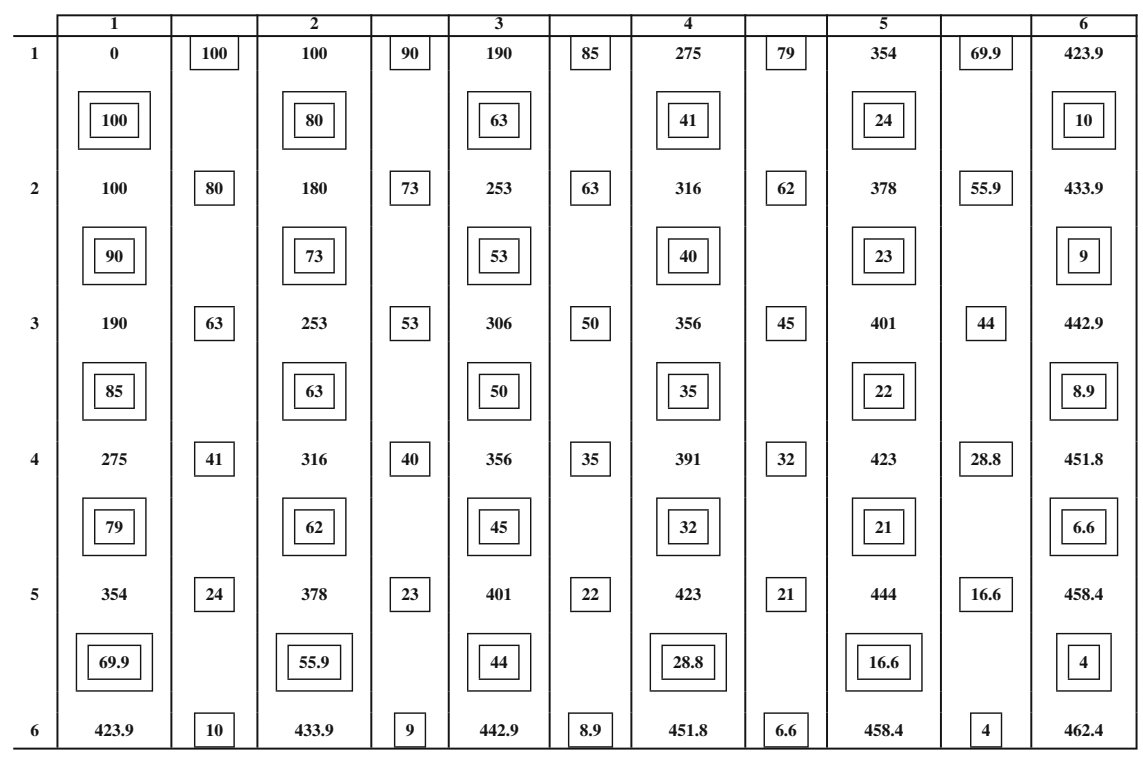

We have strict productivity, and the DMP property holds strictly. Condition H1 is met, since the SP's associated with diagonal elements are $73-63=10,50-40=10$, $32-22=10$, and 16.6-6.6 = 10. Condition H2 is met, since the SP associated with every profile above the diagonal is positive (in fact all SPs are positive). Condition H3 is met, since (for example) the SP at $(3,5)$ is $(22-9)>10$. Condition H4 is met, since $A_{26}-A_{16}=10$. We shall not present the surplus matrix, nor the payoff matrix of the sharing game in which each player receives half the revenue. But it is easy to verify that $(1,6)$ and $(6,1)$ are efficient and that the game has three (pure-strategy) equilibria: the two efficient profiles and $(5,5)$, which is squandering. (Moreover, $(5,5)$ is not Pareto-dominated by $(1,6)$ or $(6,1)$; at $(5,5)$, each player's payoff is $222-50=172$, while at the efficient pairs, one player's payoff is 201.95 and the other's payoff is 151.95).

\section{Squandering in information-gathering games}

We have just seen that squandering equilibria are not isolated phenomena but exist, generically, in certain classes of finite sharing games that display the Substitutes property sufficiently strongly. In our earlier paper, ${ }^{27}$ we found a class of games whose strategy sets are continua, which also display the Substitutes property and possess squandering equilibria. It seems intuitively plausible, moreover, that squandering equilibria exist, in certain settings, when each player's efforts are devoted to gathering

27 See Theorem 8 in Courtney and Marschak (2006). 
information about an external random variable. They report their results to a central Headquarters, which thereupon chooses an action. The organization then collects a payoff which is a function of the action chosen and the true value of the external random variable. HQ always chooses an action which maximizes the conditional expected value of payoff, given the players' reports. Our "revenue" is then the expected value, over all possible reports, of the payoff earned when HQ makes its best-action choice in response to the reports. Each player receives a share of revenue, and his net gain equals that share minus his own information-gathering cost. We have the Substitutes property if the average gain in expected payoff when a player increases his information-gathering effort, drops when some other player increases her effort. That might occur, in particular, if the players external variables are correlated. We might then have squandering relative to efficient information-gathering, i.e., the organization suffers from "information overload" - to use a popular but quite imprecise term.

In the search for such situations, it is natural to start with very simple games. We shall consider three two-player examples.

Example 1: Each player specializes in an external variable and partitions the set of its possible values. ${ }^{28}$ The organization's payoff depends on the action taken by HQ and on a regularly changing random variable $Z=\left(Z_{1}, Z_{2}\right)$, which takes 8 possible values, denoted $P_{1}, \ldots, P_{8}$, in $[0,4] \times[0,4]$. It is common knowledge that each of these eight points is equally probable. Player $i$ in $\{1,2\}$ specializes in $Z_{i}$ and his job is to learn about the current $Z_{i}$. He can learn more by spending more. He chooses an effort level $x_{i} \in\{1,2,3,4\}$, and divides the interval $[0,4]$ into $2^{x_{i}-1}$ intervals of equal length. His cost is a linear function of $x_{i}$. Having found the current interval, he reports his finding to HQ. HQ uses the two reports to choose an action $a=\left(a_{1}, a_{2}\right) \in[0,4] \times[0,4]$. The organization then earns

$$
W(Z, a)=50-20\left(\left(a_{1}-Z_{1}\right)^{2}+\left(a_{2}-Z_{2}\right)^{2}\right) .
$$

HQ chooses the action which maximizes the expected value of $W$, conditional on the reports received. Our "revenue" $A\left(x_{1}, x_{2}\right)$ is the expected value of the highest attainable conditional expected value of $W$. Each player receives half of the revenue.

The best action of HQ is easy to compute. Given the two reports, which tell HQ that $Z$ lies in some rectangle $T$, the best action $a$ is a minimizer of the average value of the squared distance $\left\|a-P_{i}\right\|^{2}$ where $i$ runs over the indices for which $P_{i} \in T$. So HQ minimizes $\sum_{P_{i} \in R}\left\|a-P_{i}\right\|^{2}$. The minimizing $a$ is the centroid of the points $P_{i} \in T$. Those points can be chosen so that the sharing game has a squandering equilibrium. That is the case, for example, if the points are:

$$
\begin{aligned}
& P_{1}=(3.3,3.9) \\
& P_{2}=(3.6,1.9) \\
& P_{3}=(1.6,2.2) \\
& P_{4}=(2.1,3.7)
\end{aligned}
$$

\footnotetext{
28 An analysis of the effect of information-technology improvement in a class of information-gathering games that are similar to this example is given in Marschak (2004).
} 


$$
\begin{aligned}
& P_{5}=(1.4,4) \\
& P_{6}=(2.6,1.2) \\
& P_{7}=(.6, .6) \\
& P_{8}=(3.8,1.9)
\end{aligned}
$$

For each player, let the cost of choosing the $j$ th action be $0.5(j-1)$ Then doing nothing (choosing a one-set partititoning) is free, splitting the interval in two costs 0.5 , splitting the interval in four costs 1 , and splitting the interval in eight costs 1.5. Suppose player 2 does nothing and player 1 chooses the finest division. We see that HQ can then locate exactly 6 of the points, and only has to guess the average of $P_{2}$ and $P_{8}$. But HQ can also do this when both players choose to divide their intervals in fourths, although that costs more.

The sharing game's revenue $A$ is shown in the following matrix. (A minus sign indicates a negative quantity approximately equal to -2 ).

$\left.\begin{array}{l|cccc} & 1 \text { ( costs zero) } & 2(\text { costs .5) } & 4 \text { (costs } 1) & 8 \text { (costs } 1.5) \\ 1 \text { (costs zero) } & - & 20.2 & 42.383 & 45.217 \\ 2 \text { (costs 0.5) } & 14.543 & 41.167 & 45.267 & 48.1 \\ 4(\operatorname{costs} 1) & 30.792 & 45.85 & 49.95 & 49.95 \\ 8(\operatorname{costs} 1.5) & 49.95 & 49.95 & 49.95 & 49.95\end{array}\right)$

The only violations of the Substitutes property occur near the effort levels $(1,1)$. Starting there, a move by either player from 1 to 2 causes the other's marginal productivity to increase. Subtracting total cost from each revenue, we find that the unique efficient pair is $(4,1)$, where total cost is 1 . The sharing game's payoff matrix is as follows. Equilibria are in boldface and a minus sign indicates a negative quantity.

1 24

8

1
2
4
8 $\left(\begin{array}{cccc}(-,-) & (10.1,9.6) & (21.1915,20.1915) & (22.6085,21.1085) \\ (6.7715,7.2715) & (20.0835,20.0835) & (22.1335,21.6335) & (23.55,22.55) \\ (\mathbf{2 3 . 4 7 5}, \mathbf{2 4 . 9 7 5}) & (23.475,24.475) & (23.475,23.975) & (23.475,23.475)\end{array}\right)$

The equilibrium $(4,4)$ has total cost 2 and is therefore squandering.

Example 2: Each player samples from the same normal distribution, which has known variance and unknown mean; the payoff function is quadratic; and HQ and the players have the same normal prior on the possible values of the unknown mean. Now suppose there is just a single external random variable $e$. Its support is the real line. It has a normal distribution with variance one and unknown mean $\mu$. HQ and the players have a common prior on the possible values of $\mu$. That prior is itself normal, with variance one and mean zero. HQ has to choose an action $a \in \mathbb{R}$. For the pair $(a, e)$, the payoff $2 e a-a^{2}$ is earned once $e$ is realized. Player $i \in\{1,2\}$ draws a sample of size $x_{i}$ from the unknown distribution. Player $i$ 's strategy set is the set of positive integers. The cost 
associated with $x_{i}$ is $k x_{i}$. HQ receives the combined sample, of size $x_{1}+x_{2}$. Denote the sample $D\left(x_{1}, x_{2}\right)$ and the sum of its elements $Y_{x_{1}+x_{2}}$. HQ then takes the action which maximizes the posterior conditional expectation $E\left(2 \mu a-a^{2} \mid D\left(x_{1}, x_{2}\right)\right)$. The maximizing action is the posterior conditional expectation $E\left(\mu \mid D\left(x_{1}, x_{2}\right)\right)$ itself. Letting $u$ denote $x_{1}+x_{2}$, we find that this posterior conditional expectation equals $\left(\frac{Y_{u}}{u+1}\right)$. The posterior expected value of $E\left(2 \mu a-a^{2} \mid D\left(x_{1}, x_{2}\right)\right)$, when the maximizing action is chosen, is $\left(\frac{Y_{u}}{u+1}\right)^{2}$. For fixed $\mu$, the expected value of $\left(\frac{Y_{u}}{u+1}\right)^{2}$ is $\frac{u+u^{2} \mu^{2}}{(u+1)^{2}}$. Using our normal prior on the possible $\mu$ s, we find that the expected value of $\frac{u+u^{2} \mu^{2}}{(u+1)^{2}}$, over all $\mu$, is $1-\frac{1}{u}=1-\frac{1}{x_{1}+x_{2}}$. That is our "revenue" $A\left(x_{1}, x_{2}\right)$, given the observing efforts $x_{1}, x_{2}$.

Note that if HQ had "perfect information", i.e., it knew the true $\mu$, then it would take the action $a=\mu$ and would achieve the expected payoff $\mu^{2}$. Taking expectations over all $\mu$, using the prior, yields a "revenue" of one. So revenue for the efforts $x_{1}, x_{2}$ approaches perfect-information revenue as the efforts grow. Now consider the sharing game in which each player receives half of revenue. That game is a symmetric Simple Substitutes Game, in the sense of Theorem B. Using the notation of that theorem, the revenue $A_{11}$ is $J=\frac{1}{2}$, and for $i+j>2$, the revenue $A_{i j}$ equals $J+\epsilon_{1}+\epsilon_{2}+\cdots+\epsilon_{i+j-2}$, where $\epsilon_{i+j-2}=\frac{1}{i+j-1}-\frac{1}{i+j}$. The epsilons are strictly decreasing, as Theorem $\mathrm{B}$ requires. Theorem $\mathrm{B}$ tells us that there is no squandering equilibrium even though we have the Substitutes property.

Example 3: There are two external variables which have a joint normal distribution with known variance/covariance matrix but unknown means; each player observes one of the variables; HQ and the players have a normal prior on the unknown means; and payoff is quadratic. Now there are two external real variables, $e_{1}, e_{2}$, distributed normally with known variance/covariance matrix $\left(\begin{array}{ll}1 & \sigma \\ \sigma & 1\end{array}\right)$ but unknown means $\mu_{1}, \mu_{2}$. HQ and the players have a normal prior on each of the unknown means. The two priors are independent. Each prior has variance 1 . The prior means are $M_{1}$ for $\mu_{1}$ and $M_{2}$ for $\mu_{2}$. HQ chooses an action pair $a=\left(a_{1}, a_{2}\right)$. After making that choice, the pair $e=\left(e_{1}, e_{2}\right)$ is realized and a payoff is earned, namely

$$
W(a, e)=2 e_{1} a_{1}+2 e_{2} a_{2}-2 q a_{1} a_{2}-a_{1}^{2}-a_{2}^{2},
$$

where $q$ is fixed and satisfies $|q|<1$. For given $e$, the unique $W$-maximizing action pair $e$ is $\hat{a}(e)=\left(\frac{e_{1}-q e_{2}}{1-q^{2}}, \frac{e_{2}-q e_{1}}{1-q^{2}}\right)$, and the highest attainable value of $W$ is

$$
W(\hat{a}(e), e)=\frac{e_{1}^{2}+e_{2}^{2}-q e_{1} e_{2}}{1-q^{2}} .
$$

Player $i \in\{1,2\}$, chooses an effort level $x_{i}$ from the nonnegative integers. Let $i^{*}$ denote a player whose effort level is highest, and let $i^{* *}$ denote the other player. Before HQ makes its action choice, at least $x_{i}$ successive pairs $e=\left(e_{1}, e_{2}\right)$ are realized. They yield information about the unknown means $\mu_{1}, \mu_{2}$. Each player $i$ observes $e_{i}$ for the 
first $x_{i * *}$ of these successive pairs. But for the next $x_{i^{*}}-x_{i^{* *}}$ pairs, Player $i^{* *}$ does no further observing, while player $i^{*}$ observes $e_{i *}$ only.

Each player reports his observations to HQ. Let $D_{i}\left(x_{i}\right)$ denote player $i$ 's report. Let $E\left(\mu_{i} \mid D_{1}\left(x_{1}\right), D_{2}\left(x_{2}\right)\right)$ denote the posterior expected value of $\mu_{i}$ given the two reports. Having received the two reports, HQ knows that for any action $a=\left(a_{1}, a_{2}\right)$, the posterior expected payoff $W\left(a,\left(E\left(\mu_{1} \mid D_{1}\left(x_{1}\right), D_{2}\left(x_{2}\right)\right), E\left(\mu_{2} \mid D_{1}\left(x_{1}\right), D_{2}\left(x_{2}\right)\right)\right)\right.$ will be earned. It takes the action which maximizes that expected payoff. The maximizing action is $\hat{a}\left(\left(E\left(\mu_{1} \mid D_{1}\left(x_{1}\right), D_{2}\left(x_{2}\right)\right), E\left(\mu_{2} \mid D_{1}\left(x_{1}\right), D_{2}\left(x_{2}\right)\right)\right)\right.$, where $\hat{a}$ is the function given above. Using $(+)$, we see that the maximizing action yields the posterior expected payoff

$$
\begin{aligned}
& W\left(\hat{a}(),\left(E\left(\mu_{1} \mid D_{1}\left(x_{1}\right), D_{2}\left(x_{2}\right)\right), E\left(\mu_{2} \mid D_{1}\left(x_{1}\right), D_{2}\left(x_{2}\right)\right)\right)\right) \\
&= \frac{1}{1-q^{2}} \cdot\left[\left(E\left(\mu_{1} \mid D_{1}\left(x_{1}\right), D_{2}\left(x_{2}\right)\right)\right)^{2}+\left(E\left(\mu_{2} \mid D_{1}\left(x_{1}\right), D_{2}\left(x_{2}\right)\right)\right)^{2}\right. \\
&\left.-q \cdot E\left(\mu_{1} \mid D_{1}\left(x_{1}\right), D_{2}\left(x_{2}\right)\right) \cdot E\left(\mu_{2} \mid D_{1}\left(x_{1}\right), D_{2}\left(x_{2}\right)\right)\right]
\end{aligned}
$$

Our "revenue" (a function of $x_{1}$ and $x_{2}$ ) is the expectation of that posterior expected payoff, over all the possible reports $D_{1}\left(x_{1}\right), D_{2}\left(x_{2}\right)$ and all the possible values of the unknown means. More explicitly, note that for fixed $x_{1}, x_{2}$, the two reports are random variables whose joint density is determined by the unknown means. The same is true of the joint density of the posterior expected values of the means given the two reports, since those posterior expected values are functions of the two reports. For given $x_{1}, x_{2}$, let $H_{i}\left(x_{1}, x_{2} ; \tilde{\mu}_{1}, \tilde{\mu}_{2}\right)$ denote the expected value, over all reports $D_{1}\left(x_{1}\right), D_{2}\left(x_{2}\right)$, of $E\left(\mu_{i} \mid D_{1}\left(x_{1}\right), D_{2}\left(x_{2}\right)\right)$, when the true means are $\tilde{\mu}_{1}, \tilde{\mu}_{2}$. Let the symbol $\mathcal{E}$ denote an expectation that is taken with respect to the pairs $\left(\tilde{\mu}_{1}, \tilde{\mu}_{2}\right)$, using the prior densities on $\tilde{\mu}_{1}, \tilde{\mu}_{2}$ that are defined by the means $M_{1}, M_{2}$. Then our revenue is

$$
\begin{aligned}
A\left(x_{1}, x_{2}\right)= & \frac{1}{1-q^{2}} \cdot\left(\mathcal { E } \left[\left(H_{1}\left(x_{1}, x_{2} ; \tilde{\mu}_{1}, \tilde{\mu}_{2}\right)^{2}\right]+\mathcal{E}\left[\left(H_{2}\left(x_{1}, x_{2} ; \tilde{\mu}_{1}, \tilde{\mu}_{2}\right)^{2}\right]\right.\right.\right. \\
& \left.-q \cdot \mathcal{E}\left[H_{1}\left(x_{1}, x_{2} ; \tilde{\mu}_{1}, \tilde{\mu}_{2}\right) \cdot H_{2}\left(x_{1}, x_{2} ; \tilde{\mu}_{1}, \tilde{\mu}_{2}\right)\right]\right) .
\end{aligned}
$$

Under "perfect information", HQ knows $\mu_{1}$ and $\mu_{2}$ exactly. As we increase $x_{1}$ and $x_{2}$, our revenue $A$ gets arbitrarily close to its perfect-information value. Using that fact, one can show that there exists an integer $J>0$, and a quadruple $\left(M_{1}, M_{2}, q, \sigma\right)$, such that $A$ has the DMP and Substitutes properties for all $\left(x_{1}, x_{2}\right) \in\{0,1,2, \ldots, J\} \times$ $\{0,1,2, \ldots, J\}$. Now consider the game defined by the sextuple $\left(M_{1}, M_{2}, q, \sigma, J, k\right)$, in which each player's strategy set is $\{0,1, \ldots, J\}$, each $c_{i}\left(x_{i}\right)$ equals $k x_{i}$ (where $k>0$ ), and each player receives half of revenue. Can we find a sextuple such that this game is not a Simple Substitutes Game and possesses a squandering equilibrium? The question remains open.

Clearly a great deal remains to be learned about information-gathering games that have the Substitutes property, and the conditions under which they possess squandering equilibria. One approach would be to use Theorem $\mathrm{C}$, and to try to construct information-gathering games that have the properties assumed in that theorem. 


\section{Choosing the reward functions}

Suppose the strategy sets $S_{1}, \ldots, S_{n}$, the revenue function $A$, and the cost functions $c_{1}, \ldots, c_{n}$ are fixed. Consider the following quest: for an interesting class of reward functions-but not collective-punishment functions-find those in which surplus is high at some (or all) equilibria. To start, consider the large class of NDR functions. The following general theorem compares an equilibrium of an NDR game with an equilibrium of another game, which need not have the NDR property. It says that if players work harder at the first of those two equilibria, then surplus must be higher at the first equilibrium as well. We let $\sigma(x)$ denote the surplus $A(x)-\sum_{i \in N} c_{i}\left(x_{i}\right)$.

Theorem D: For the triple $\left(A,\left\{S_{i}\right\}_{i \in N},\left\{c_{i}\right\}_{i \in N}\right)$, suppose the strategy profile $x^{\prime}$ is an equilibrium of the productive sharing game

$$
G^{\prime}=\left\langle A,\left\{S_{i}\right\}_{i \in N},\left\{c_{i}\right\}_{i \in N},\left\{\rho_{i}^{\prime}\right\}_{i \in N}\right\rangle
$$

and $x^{\prime \prime}$ is an equilibrium of the productive sharing game

$$
G^{\prime \prime}=\left\langle A,\left\{S_{i}\right\}_{i \in N},\left\{c_{i}\right\}_{i \in N},\left\{\rho_{i}^{\prime \prime}\right\}_{i \in N}\right\rangle,
$$

where each $\rho_{i}^{\prime}$ and each $\rho_{i}^{\prime \prime}$ is nondecreasing, and $\left\{\rho_{i}^{\prime \prime}\right\}_{i \in N}$ has the NDR property. Suppose we have

$$
c_{i}\left(x_{i}^{\prime \prime}\right) \geq c_{i}\left(x_{i}^{\prime}\right) \text { for all } i .
$$

Then $\sigma\left(x^{\prime \prime}\right) \geq \sigma\left(x^{\prime}\right)$. That inequality is strict if $(i)$ the game is strictly productive and (ii) for some $i$ the inequality in $(+)$ is strict and $\rho_{i}^{\prime \prime}$ is strictly increasing.

Proof: Consider any player $i$. In the game $G^{\prime \prime}$, player $i$ does not want to deviate from the equilibrium $x^{\prime \prime}$ to the strategy $x_{i}^{\prime}$, i.e., using a standard notation,

$$
\rho_{i}^{\prime \prime}\left(A\left(x_{i}^{\prime}, x_{-i}^{\prime \prime}\right)\right)-c_{i}\left(x^{\prime}\right) \leq \rho_{i}^{\prime \prime}\left(A\left(x^{\prime \prime}\right)\right)-c_{i}\left(x^{\prime \prime}\right) \text {. }
$$

Condition (+), productivity, and the fact that $\rho_{i}^{\prime \prime}$ is nondecreasing imply $\rho_{i}^{\prime \prime}\left(A\left(x^{\prime}\right)\right) \leq$ $\rho_{i}^{\prime \prime}\left(A\left(x_{i}^{\prime}, x_{-i}^{\prime \prime}\right)\right)$. That, together with $(*)$, implies

$$
\rho_{i}^{\prime \prime}\left(A\left(x^{\prime}\right)\right)-c_{i}\left(x^{\prime}\right) \leq \rho_{i}^{\prime \prime}\left(A\left(x^{\prime \prime}\right)\right)-c_{i}\left(x^{\prime \prime}\right) .
$$

Rearranging and summing over all $i$, we obtain

$$
\sum_{i \in N} c_{i}\left(x_{i}^{\prime \prime}\right)-\sum_{i \in N} c_{i}\left(x_{i}^{\prime}\right) \leq \sum_{i \in N} \rho_{i}^{\prime \prime}\left(A\left(x^{\prime \prime}\right)\right)-\sum_{i \in N} \rho_{i}^{\prime \prime}\left(A\left(x^{\prime}\right)\right) .
$$

Since (by (+) and productivity) $A\left(x^{\prime \prime}\right) \geq A\left(x^{\prime}\right)$, and since $\left\{\rho_{i}^{\prime \prime}\right\}_{i \in N}$ has the NDR property, we have $A\left(x^{\prime}\right)-\sum_{i \in N} \rho_{i}^{\prime \prime}\left(A\left(x^{\prime}\right)\right) \leq A\left(x^{\prime \prime}\right)-\sum_{i \in N} \rho_{i}^{\prime \prime}\left(A\left(x^{\prime \prime}\right)\right)$ or

$$
\sum_{i \in N} \rho_{i}^{\prime \prime}\left(A\left(x^{\prime \prime}\right)\right)-\sum_{i \in N} \rho_{i}^{\prime \prime}\left(A\left(x^{\prime}\right)\right) \leq A\left(x^{\prime \prime}\right)-A\left(x^{\prime}\right) .
$$


That, together with $(* * *)$ imply

$$
\sum_{i \in N} c_{i}\left(x_{i}^{\prime \prime}\right)-\sum_{i \in N} c_{i}\left(x_{i}^{\prime}\right) \leq A\left(x^{\prime \prime}\right)-A\left(x^{\prime}\right) .
$$

Rearranging, we obtain

$$
\sigma\left(x^{\prime \prime}\right) \geq \sigma\left(x^{\prime}\right)
$$

as claimed. If the game is strictly productive, and for some $i, \rho_{i}^{\prime \prime}$ is strictly increasing and we have $c_{i}\left(x_{i}^{\prime \prime}\right)>c_{i}\left(x_{i}^{\prime}\right)$, then for that $i$, the inequality $(* *)$ is strict. Hence $(* * *)$ is strict and therefore $\sigma\left(x^{\prime \prime}\right)>\sigma\left(x^{\prime}\right)$, as claimed.

Informally speaking, it is socially desirable for the parties concerned to agree on a reward-function profile that induces the agents to work hard (at equilibrium). We now use Theorem D in studying the choice of reward functions in the principal/agents setting. We are interested in the principal's preferences on a class of reward functions, the agents' (players') preferences, and the society's preferences (surplus). First we have a simple but general theorem about players' preferences. It has the same form as Theorem D, which dealt with society's preferences. Again, the triple $\left(A,\left\{S_{i}\right\}_{i \in N},\left\{c_{i}\right\}_{i \in N}\right)$ is fixed. Strict productivity is assumed. The theorem says that given two games that differ with regard to reward functions, where the second game's functions are at least as generous as those of the first game, every player finds an equilibrium of the second to be at least as good as an equilibrium of the first if every player works at least as hard at the second equilibrium, and some player prefers the second equilibrium if at least one player works harder.

Theorem E: Suppose the strategy profile $x^{\prime}$ is an equilibrium of the strictly productive sharing game $G^{\prime}=\left\langle A,\left\{S_{i}\right\}_{i \in N},\left\{c_{i}\right\}_{i \in N},\left\{\rho_{i}^{\prime}\right\}_{i \in N}\right\rangle$ and $x^{\prime \prime}$ is an equilibrium of the strictly productive sharing game $G^{\prime \prime}=\left\langle A,\left\{S_{i}\right\}_{i \in N},\left\{c_{i}\right\}_{i \in N},\left\{\rho_{i}^{\prime \prime}\right\}_{i \in N}\right\rangle$, where each $\rho_{i}^{\prime}$ and each $\rho_{i}^{\prime \prime}$ is strictly increasing. Suppose that for all $i$ we have

$$
c_{i}\left(x_{i}^{\prime \prime}\right) \geq c_{i}\left(x_{i}^{\prime}\right)
$$

Suppose that for every $z \in A(S)$ and every $i$, we have $\rho_{i}^{\prime \prime}(z) \geq \rho_{i}^{\prime}(z)$. Then for every player $i$, we have $\rho_{i}^{\prime \prime}\left(A\left(x^{\prime \prime}\right)\right)-c_{i}\left(x_{i}^{\prime \prime}\right) \geq \rho_{i}^{\prime}\left(A\left(x^{\prime}\right)\right)-c_{i}\left(x_{i}^{\prime}\right)$, and that inequality is strict for every $i$ if the inequality in $(+)$ is strict for some $i$.

Proof: Productivity, our assumption on the $\rho_{i}^{\prime \prime}$ and the $\rho_{i}^{\prime}$, and our assumption (+) imply that for every $i$ we have

$$
\rho_{i}^{\prime}\left(A\left(x_{i}^{\prime}, x_{-i}^{\prime}\right)\right)-c_{i}\left(x_{i}^{\prime}\right) \leq \rho_{i}^{\prime}\left(A\left(x_{i}^{\prime}, x_{-i}^{\prime \prime}\right)\right)-c_{i}\left(x_{i}^{\prime}\right) \leq \rho_{i}^{\prime \prime}\left(A\left(x_{i}^{\prime}, x_{-i}^{\prime \prime}\right)\right)-c_{i}\left(x_{i}^{\prime}\right) .
$$

Since, in the game $G^{\prime \prime}$, the strategy $x_{i}^{\prime \prime}$ is a best reply for $i$ to $x_{-i}^{\prime \prime}$, we have

$$
\rho_{i}^{\prime \prime}\left(A\left(x_{i}^{\prime}, x_{-i}^{\prime \prime}\right)\right)-c_{i}\left(x_{i}^{\prime}\right) \leq \rho_{i}^{\prime \prime}\left(A\left(x_{i}^{\prime \prime}, x_{-i}^{\prime \prime}\right)\right)-c_{i}\left(x_{i}^{\prime \prime}\right) .
$$


Combining that with the inequalities preceding it, we obtain

$$
\rho_{i}^{\prime}\left(A\left(x^{\prime}\right)\right)-c_{i}\left(x_{i}^{\prime}\right) \leq \rho_{i}^{\prime \prime}\left(A\left(x^{\prime \prime}\right)\right)-c_{i}\left(x_{i}^{\prime \prime}\right),
$$

as claimed. If we have $c_{i}\left(x_{i}^{\prime \prime}\right)>c_{i}\left(x_{i}^{\prime}\right)$ for some $i$, then $A\left(x^{\prime \prime}\right)>A\left(x^{\prime}\right)$ and hence the last inequality in $(*)$ is strict. Therefore the inequality in $(* *)$ is strict, as claimed.

Theorem D tells us that "society" prefers an equilibrium at which players work harder,and Theorem E tells us that the players prefer the harder-working equilibrium as well, provided it is an equilibrium of a game whose reward functions are more generous. We now ask: when is it the case that more generous rewards indeed induce the players to work harder at equilibrium? Here complementarity plays a crucial role. We confine attention to effort games with linearly related rewards. (Recall that the effort-game property means that a strategy is uniquely defined by its cost). Given the triple $\left(\left\{S_{i}\right\}_{i \in N}, A,\left\{c_{i}\right\}_{i \in N}\right)$, player $i$ 's net payoff at the profile $x=\left(x_{1}, \ldots, x_{n}\right)$ is determined by an increasing function $\rho: \mathbb{R} \rightarrow \mathbb{R}^{+}$and a positive number $r_{i}$. Let $\Lambda$ denote the vector $\left(r_{1}, \ldots, r_{n}\right)$. Player $i$ 's net payoff is

$$
f_{\Lambda}^{i}(x)=r_{i} \cdot \rho(A(x))-c_{i}\left(x_{i}\right)
$$

Suppose the composition of $\rho$ and $A$ has the complementarity property. ${ }^{29}$ Thus

$$
\begin{aligned}
x_{i}^{\prime \prime} & \geq x_{i}^{\prime}, x_{-i}^{\prime \prime} \geq x_{-i}^{\prime} \text { implies } \rho\left(A\left(x_{i}^{\prime \prime}, x_{-i}^{\prime \prime}\right)\right)-\rho\left(A\left(x_{i}^{\prime}, x_{-i}^{\prime \prime}\right)\right) \\
& \geq \rho\left(A\left(x_{i}^{\prime \prime}, x_{-i}^{\prime}\right)\right)-\rho\left(A\left(x_{i}^{\prime}, x_{-i}^{\prime}\right)\right) .
\end{aligned}
$$

We then have (in the terminology of the supermodularity literature) the following two "increasing differences" statements:

$$
\begin{aligned}
& \text { for any fixed } x_{-i}, f_{\Lambda^{\prime \prime}}^{i}\left(x_{i}^{\prime \prime}, x_{-i}\right)-f_{\Lambda^{\prime \prime}}^{i}\left(x_{i}^{\prime}, x_{-i}\right) \geq f_{\Lambda^{\prime}}^{i}\left(x_{i}^{\prime \prime}, x_{-i}\right)-f_{\Lambda^{\prime}}^{i}\left(x_{i}^{\prime}, x_{-i}\right) \\
& \qquad \text { if } x_{i}^{\prime \prime} \geq x_{i}^{\prime} \text { and } \Lambda^{\prime \prime} \geq \Lambda^{\prime} . \\
& \text { for any fixed } \Lambda, f_{\Lambda}^{i}\left(x_{i}^{\prime \prime}, x_{-i}^{\prime \prime}\right)-f_{\Lambda}^{i}\left(x_{i}^{\prime}, x_{-i}^{\prime \prime}\right) \geq f_{\Lambda}^{i}\left(x_{i}^{\prime \prime}, x_{-i}^{\prime}\right)-f_{\Lambda}^{i}\left(x_{i}^{\prime}, x_{-i}^{\prime}\right) \\
& \quad \text { if } x_{i}^{\prime \prime} \geq x_{i}^{\prime} \text { and } x_{-i}^{\prime \prime} \geq x_{-i}^{\prime} .
\end{aligned}
$$

In view of these statements, we can directly apply results from the supermodulargames literature, for example, Theorem 4.2.2 in Topkis (1998). ${ }^{30}$ We then obtain the following theorem.

Theorem F: Fix the triple $\left(\left\{S_{i}\right\}_{i \in N}, A,\left\{c_{i}\right\}_{i \in N}\right)$, where, for each $i, S_{i} \subseteq \mathbb{R}$ and $S_{i}$ is compact. Let the triple have the effort-game property. Fix the increasing function

\footnotetext{
29 We shall let a statement like " $w^{\prime} \geq w$ ", where $w=\left(w_{1}, \ldots, w_{t}\right)$ and $w^{\prime}=\left(w_{1}^{\prime}, \ldots, w_{t}^{\prime}\right)$, mean that $w_{i}^{\prime} \geq w_{i}$ for all $i \in\{1, \ldots, t\}$. A vector $w$ is greatest (least) in a given set if $w \geq(\leq) w^{\prime}$ for every $w^{\prime}$ in the set.

30 That theorem is stronger than needed for our purposes, since it allows each player's strategy set to lack a complete ordering. In our effort-game case, each strategy set is completely ordered by cost (i.e., the set is a "chain").
} 
$\rho: \mathbb{R} \rightarrow \mathbb{R}^{+}$. For every $\Lambda=\left(r_{1}, \ldots, r_{n}\right) \in \mathbb{R}^{n^{+}}$, consider the strictly productive effort game $G_{\Lambda}=\left\langle\left\{S_{i}\right\}_{i \in N}, A,\left\{c_{i}\right\}_{i \in N},\left\{r_{i} \rho\right\}_{i \in N}\right\rangle$, where the function $\rho \circ A$ (the composition of $A$ and $\rho$ ) $i$ is upper semi-continuous and has the complementarity property. Then (i) $G_{\Lambda}$ has a greatest equilibrium $x_{\Lambda}$ and a least equilibrium $x_{\Lambda}^{*}$; (ii) if $\Lambda^{\prime \prime} \geq \Lambda^{\prime}$, then $x_{\Lambda^{\prime \prime}} \geq x_{\Lambda^{\prime}}$ and $x_{\Lambda^{\prime \prime}}^{*} \geq x_{\Lambda^{\prime}}^{*}$.

So we can select an equilibrium for each $\Lambda$ in such a way that if some player's reward parameter $r_{i}$ goes up, then at the new equilibrium no player works less hard than at the old one. If we place further conditions on $A$ and the $c_{i}$, then at least one player works harder at the new equilibrium.

Finally, consider a principal and his agents, who are prospective sharing-game players. They negotiate about the game's rewards. In the following simple example, where $n=2$ and every $\Lambda$ takes the form $(r, r)$, we have a strong conclusion.

For each of the two players the strategy set is $\mathbb{R}^{+}$. The revenue function is $A\left(x_{1}, x_{2}\right)$ $=\sqrt{x_{1} x_{2}}$. Each player's reward is $r \cdot A$, where $0 \leq r \leq \frac{1}{2}$. The cost functions are $c_{i}\left(x_{i}\right)=x_{i}^{2}, i=1,2$. We have complementarity, since $\frac{\partial^{2} A}{\partial x_{1} \partial x_{2}}>0$. Given that Player 2 has chosen $x_{2}$, Player 1's payoff for a given $x_{1}$ is $r \cdot \sqrt{x_{1} x_{2}}-x_{1}^{2}$. The second derivative of this payoff with respect to $x_{1}$ is negative. So Player 1's best reply to a positive $x_{2}$ is the solution of the first-order condition

$$
\frac{r}{2 \sqrt{x_{1} x_{2}}} \cdot x_{2}=2 x_{1} \text {. }
$$

There is an analagous statement for Player 2. Thus we have an equilibrium at $\left(x_{1}, x_{2}\right)$, where $x_{1}>0, x_{2}>0$, if and only if

$$
\frac{r}{2 \sqrt{x_{1} x_{2}}} \cdot x_{2}=2 x_{1}, \quad \frac{r}{2 \sqrt{x_{1} x_{2}}} \cdot x_{1}=2 x_{2} \text {. }
$$

That implies ${ }^{31} x_{1}=x_{2}$. Letting $z$ denote the common value of both player's strategies at the equilibrium, we obtain $\frac{r}{2 \sqrt{z \cdot z}}=2 z$, or $z=\frac{r}{4}$. Thus the unique interior equilibrium is $\left(\frac{r}{4}, \frac{r}{4}\right)$. At that equilibrium, each player's payoff is $\frac{3 r^{2}}{16}$. There is another equilibrium at $(0,0)$, but both players prefer $\left(\frac{r}{4}, \frac{r}{4}\right)$. At the equilibrium $\left(\frac{r}{4}, \frac{r}{4}\right)$, revenue is $\sqrt{\frac{r}{4} \cdot \frac{r}{4}}=\frac{r}{4}$, and residual is

$$
(1-2 r) \cdot \frac{r}{4}=\frac{r}{4}-\frac{r^{2}}{2} .
$$

The derivative of residual is $\frac{1}{4}-r$ and the second derivative is -1 . Thus residual is zero at $r=0$, rises to a peak of $\frac{1}{32}$ at $r=\frac{1}{4}$, and then drops, reaching zero again at $r=\frac{1}{2}$. The principal's favorite $r$ is $\frac{1}{4}$. Each player's payoff at the equilibrium $\left(\frac{r}{4}, \frac{r}{4}\right)$ is

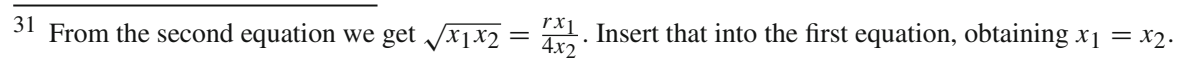




$$
r \cdot \sqrt{\frac{r}{4} \cdot \frac{r}{4}}-\left(\frac{r}{4}\right)^{2}=\frac{3 r^{2}}{16} .
$$

So for $0 \leq r^{\prime}<r^{\prime \prime}<\frac{1}{4}$, all parties prefer $r^{\prime \prime}$ to $r^{\prime}$, but for $\frac{1}{4} \leq r^{\prime}<r^{\prime \prime} \leq \frac{1}{2}$, the principal prefers $r^{\prime}$ to $r^{\prime \prime}$ and the players prefer $r^{\prime \prime}$ to $r^{\prime}$. The negotiation set is the interval $\left[\frac{1}{4}, \frac{1}{2}\right]$. The further towards the upper bound of $\frac{1}{2}$ the players are able to push the final bargain, the harder they work at equilibrium, and hence the larger-according to Theorem $\mathrm{E}$ - is the resulting surplus.

Can this strong conclusion be generalized? Let us continue to consider the case of two players who have the same reward parameter $r$. Complementarity of $A$ does not, in general, guarantee that the principal's residual will be a single-peaked function of $r$, as it was in the preceding example. To see this, consider the following example:

$$
\begin{aligned}
& S_{1}=S_{2}=[32 / 45, \infty] ; c_{i}\left(x_{i}\right)=(15 / 2) x_{i}^{4}-(32 / 3) x_{i}^{3}+(3 / 2) x_{i}^{2}, i=1,2 ; \\
& \quad A\left(x_{1}, x_{2}\right)=A_{1}\left(x_{1}\right)+A_{2}\left(x_{2}\right), \text { where } A_{i}\left(x_{i}\right)=\left(30 x_{i}^{3}-16 x_{i}^{2}+3 x_{i}\right), i=1,2 .
\end{aligned}
$$

Here complementarity holds trivially since $A$ is additively separable. On the domain $S_{i}$, the function $c_{i}$ is strictly increasing, and we have strict productivity on $S_{1} \times S_{2}$. Again, $r$ is to be chosen from the interval $\left[0, \frac{1}{2}\right]$. On each $S_{i}$, player $i$ 's payoff for a given positive choice of the other player is $r A_{i}\left(x_{i}\right)-c_{i}\left(x_{i}\right)$. That has negative second derivative on $S_{i}$ for $r \in\left[0, \frac{1}{2}\right]$. Player $i$ 's best reply to any positive choice of the other player is then the unique solution to the first-order condition $r A_{i}^{\prime}\left(x_{i}\right)-c_{i}^{\prime}\left(x_{i}\right)=0$ and that solution is quickly seen to be $x_{i}=r$. So $(r, r)$ is the unique equilibrium. At that equilibrium, the principal's residual is $(1-2 r)(2) A_{1}(r)=(2-4 r)\left(30 r^{3}-16 r^{2}+3 r\right)$. That again equals zero at $r=0$ and $r=\frac{1}{2}$, but it does not have a single peak, as the previous example did. Instead it rises and falls several times between zero and $\frac{1}{2}$. Nevertheless, there is a value of $r$, say $r^{*}$, with $0<r^{*}<\frac{1}{2}$ such that residual falls in the interval $\left[r^{*}, \frac{1}{2}\right]$. Moreover, we may apply Theorem F (and we can also check directly) to conclude that in the interval $\left[r^{*}, \frac{1}{2}\right]$ players prefer the higher of two given values of $r$. So we find that the negotiation set contains the interval $\left[r^{*}, \frac{1}{2}\right]$. In view of Theorem F, it is in the social interest for the negotiated $r$ to be as close as possible to $\frac{1}{2}$.

We can generalize this example. Specifically, we can show that such an interval $\left[r^{*}, \frac{1}{2}\right]$ exists if we make appropriate differentiability assumptions. In fact we now show the existence of such an interval in a more general setting, where the individual players' rewards are allowed to differ from each other.

Suppose each player's strategy set consists of a closed real interval. The agents (players) start negotiations with the principal after having settled differences among themselves, so that they become a single unified bargainer. Specifically they have chosen a base vector $r^{*}=\left(r_{1}^{*}, \ldots, r_{n}^{*}\right)$, with all its elements positive. (The simplest example is $r^{*}=(1,1, \ldots, 1)$, which we just considered for the case $\left.n=2\right)$. The negotiations with the principal concern the selection of a positive real number $\gamma>0$ so that for the revenue $z$, player $i$ 's reward is $\gamma r_{i}^{*} z$ and the principal's residual is $z\left(1-\gamma \sum_{i \in N} r_{i}^{*}\right)$. Once $\gamma$ has been chosen, the game $G(\gamma)=$ $\left\langle A,\left\{S_{i}\right\}_{i \in N},\left\{c_{i}\right\}_{i \in N},\left\{\gamma r_{i}^{*}\right\}_{i \in N}\right\rangle$ will be played. At $\gamma=0$ the only equilibrium is 
$(0,0, \ldots, 0)$ and for that equilibrium residual is zero. Residual is also zero at $\gamma=$ $1 /\left(\sum_{i \in N} r_{i}^{*}\right)$. The principal insists that residual be nonnegative. So the negotiation will select a value of $\gamma$ in the interval $\left[0,1 /\left(\sum_{i \in N} r_{i}^{*}\right)\right]$. The agents agree among themselves that they prefer $\gamma^{\prime}$ to $\gamma^{\prime \prime}$ if and only if the sum of the $n$ net payoffs (rewards minus costs) at the greatest equilibrium of the game $G\left(\gamma^{\prime}\right)$ exceeds the sum of the $n$ net payoffs at the greatest equilibrium of the game $G\left(\gamma^{\prime \prime}\right)$. The principal prefers $\gamma^{\prime}$ to $\gamma^{\prime \prime}$ if and only if his residual at the greatest equilibrium of the game $G\left(\gamma^{\prime}\right)$ exceeds his residual at the greatest equilibrium of the game $G\left(\gamma^{\prime \prime}\right)$.

For this class of games, Theorem $\mathrm{F}$ implies the following theorem.

Theorem G: Fix a vector $r=\left(r_{1}, \ldots, r_{n}\right)$, where $0 \leq r_{j} \leq 1$ for all $j$, and $r_{j}$ is positive for at least one $j$. Fix the triple $\left(\left\{S_{i}\right\}_{i \in N}, A,\left\{c_{i}\right\}_{i \in N}\right)$, where $S_{i}$ is a closed interval for each $i$, the triple has the effort-game property, $A$ is everywhere positive, and complementarity holds. Consider the strictly productive games $G(\gamma)=\left\langle A,\left\{S_{i}\right\}_{i \in N},\left\{c_{i}\right\}_{i \in N},\left\{\gamma r_{i}\right\}_{i \in N}\right\rangle$ where $\gamma \in\left[0,\left(\sum_{i \in N} r_{i}\right)^{-1}\right]$. Suppose that for each $\gamma$ the greatest equilibrium $x(\gamma)=\left(x_{1}(\gamma), x_{2}(\gamma), \ldots, x_{N}(\gamma)\right)$ of $G(\gamma)$, given by Theorem $F$, is in the interior of $S$, and that all functions $\left(x_{i}\right)_{i \in N},\left(c_{i}\right)_{i \in N}$, and $A$ are continuously differentiable. Then there exists $\bar{\gamma} \in\left[0,\left(\sum_{i \in N} r_{i}\right)^{-1}\right]$ such that for $\bar{\gamma}<\gamma^{\prime}<\gamma^{\prime \prime}<\left(\sum_{i \in N} r_{i}\right)^{-1}$, the sum of the players' net payoffs is higher at $x\left(\gamma^{\prime \prime}\right)$ than at $x\left(\gamma^{\prime}\right)$, while the principal's residual is lower at $x\left(\gamma^{\prime \prime}\right)$ than at $x\left(\gamma^{\prime}\right)$.

\section{Proof: See Appendix.}

The theorem tells us that both "society" and the agents (players) want $\gamma$ to be as close to its upper bound as possible. It is in the "social" interest for the agents to be strong bargainers when they confront the principal.

\section{Concluding remarks}

We have seen that two key assumptions are extremely fruitful in the theory of sharing games: complementarity of the revenue function and the NDR property for the rewards. Then no one squanders at an inefficient equilibrium, and when reward functions are open to choice, we have strong results about the interests of society, of a principal, and of the game's players. But there are games with plausible reward and cost functions and compelling squandering equilibria. Those equilibria are important when we try to trace the influence of technological improvement on the relative performance of sharing games compared to extreme centralization. We have seen that for smooth NDR games, the decentralization penalty associated with such equilibria drops when technology improves. We also saw that in a class of finite two-player games, squandering equilibria are implied when the Substitutes property holds sufficiently strongly. Enlarging that class remains a challenge, and so does the exploration of squandering in information-gathering games that have the Substitutes property.

It is natural to seek a link between sharing games and the enormous design literature that flowed from the work of Leonid Hurwicz. We note that many NDR reward functions can be defined without any knowledge of the cost functions $c_{i}$. (That is true for constant-share reward functions, for example; it is not true for collective-punishment functions). That property is attractive when cost functions are complicated, 
randomly changing, and privately known. One could formally pose the following question: given a set of acceptable surpluses, and a set of possible $n$-player cost-function profiles, what are the informationally cheapest "game forms" whose equilibria yield an acceptable surplus for every cost-function profile? Suppose the revenue function is common knowledge and so is a prior probability distribution on the possible costfunction profiles. Once the cost-function profile is realized, only Player $i$ knows the function $c_{i}$. A game form would specify (i) a set of possible strategies for each player, where $i$ 's strategy selects, for every possible $c_{i}$, both an effort level and a message that conveys information about $c_{i}$, and (ii) an outcome function which assigns to the players' chosen messages the reward functions to be used. At an equilibrium each player is content with his strategy, given the others' strategies, because it maximizes his expected payoff. An informational cost measure for the game form would be needed, perhaps some version of message-space size. If the acceptable-surplus set is large, then game forms that require (at equilibrium) complete transmission of the privately known cost functions would be needlessly expensive. The winner might be a game form whose equilibria have no transmissions at all. In such an equilibrium, the same reward functions are always used, and they do not depend on the players' privately known costs.

\section{Appendix}

Proof of Theorem B: First consider the change in surplus when we move from any profile $(i, j)$ to any profile that is not in the same antidiagonal as $(i, j)$. Either revenue goes up from $J+\epsilon_{1}+\cdots+\epsilon_{i+j-2}$ to, say, $J+\epsilon_{1}+\cdots+\epsilon_{i+j-2}+\cdots+\epsilon_{i+j-2+h}$ at an extra cost of $h k$; or revenue goes down to, say, $J+\epsilon_{1}+\cdots+\epsilon_{i+j+2-s}$ with a cost saving of $s k$. The fact that the epsilon sequence is strictly decreasing implies that if there is a drop in surplus when we make a movement of the first kind for $h=1$, then there is, a fortiori, a drop in surplus when we make a movement of the first kind for any $h$ greater than one. Similarly if there is a drop in surplus when we make a movement of the second kind for $s=1$, then there is, a fortiori, a drop in surplus when we make a movement of the second kind for any $s$ greater than one.

Moreover, if $(i, j)$ is efficient, then, in particular, surplus is not increased by making a movement of the first kind with $h=1$ or making a movement of the second kind with $s=1$. So we conclude:

$$
(i, j) \text { is efficient if and only if } \epsilon_{i+j-1} \leq k \leq \epsilon_{i+j-2} .
$$

Next note that at any profile $(i, j)$, player 1's reward loss due to replacing $i$ by $i-1$ is $\frac{1}{2} \epsilon_{i+j-2}$. If that reward loss equals or exceeds player 1's cost saving of $k$, then, a fortiori, the reward loss due to replacing $i$ by $i-t$, where $t>1$, exceeds player 1 's cost saving of $k t$. Again, that is the case because the epsilon sequence is strictly decreasing.

Similarly note that at any profile $(i, j)$, player 1 's reward gain due to replacing $i$ by $i+1$ is $\frac{1}{2} \epsilon_{i+j-1}$. If that reward gain is less than or equal to player 1's extra cost $k$, then, a fortiori the reward gain due to replacing $i$ by $i+t$, for $t>1$, is less than player 1's extra cost $k t$. 
Moreover, if $(i, j)$ is an equilibrium, then, in particular, 1's net payoff (reward minus cost) does not rise when we replace $i$ by $i+1$, and it does not rise when we replace $i$ by $i-1$.

Analagous statements hold for the deviations of player 2. So we conclude

$$
(i, j) \text { is an equilibrium if and only if } \epsilon_{i+j-1} \leq 2 k \leq \epsilon_{i+j-2} \text {. }
$$

Now let $\left(i^{*}, j^{*}\right)$ be an inefficient equilibrium and let $(\bar{i}, \bar{j})$ be efficient. Suppose that, contrary to our claim, $\left(i^{*}, j^{*}\right)$ is squandering relative to $(\bar{i}, \bar{j})$, i.e., $i^{*}+j^{*}>\bar{i}+\bar{j}$. Hence $i^{*}+j^{*}-1>\bar{i}+\bar{j}-1$ and therefore

$$
\epsilon_{i^{*}+j^{*}-1}<\epsilon_{\bar{i}+\bar{j}-1}
$$

Using (i) and the efficiency of $(\bar{i}, \bar{j})$, as well as (ii) and the fact that $\left(i^{*}, j^{*}\right)$ is an equilibrium, we now obtain (in view of (iii)):

$$
\epsilon_{i *+j^{*}-1}<\epsilon_{\bar{i}+\bar{j}-1} \leq k<2 k \leq \epsilon_{i^{*}+j^{*}-2} .
$$

But then the sufficiency part of (i) implies that $\left(i^{*}, j^{*}\right)$ is efficient, contrary to our assumption.

Proof of Theorem $C$ : It will be convenient to denote the revenue at $(i, j)$ by $A(i, j)$ rather than $A_{i j}$ (as in the text). The proof has two parts.

Part 1. Here we prove that for a productive game satisfying DMP and Conditions $\mathrm{H} 1-\mathrm{H} 4$, the profile $(1, t)$ is efficient. To do so we first establish three Claims.

Claim 1: For all $(i, j)$ satisfying $1<i \leq j<t$, we have

$$
A(i-1, j+1)-A(i, j) \geq k
$$

We can interpret the claim in terms of the antidiagonals. Recall that for a given constant $V$, with $1 \leq V \leq 2 t-1$, the " $V$ th antidiagonal" is the set of pairs $\{(i, j) \in$ $\{1,2, \ldots, t\} \times\{1,2, \ldots, t\}: i+j=V\}$. Claim 1 says that if we start at a pair $\bar{i}, \bar{j}$ with $1<\bar{i} \leq \bar{j}<t$, and we proceed along the $(\bar{i}+\bar{j})$ th antidiagonal, in the direction of increasing $j$ (and decreasing $i$ ), we find that at each step, revenue, and hence surplus, increase by at least $k$.

We shall prove the statement that for all positive integers $p$, we have $A(\ell-p$, $\ell+1)-A(\ell-p+1, \ell) \geq k$ whenever $p<\ell<t$. That is equivalent to the Claim, and we establish it by induction on $p$. By H1, the $\mathrm{SP}$ at $(\ell, \ell)$ is $\geq k$ whenever $1<\ell<t$. Therefore

$$
\begin{aligned}
& (A(\ell+1, \ell)-A(\ell, \ell))-(A(\ell, \ell+1)-A(\ell-1, \ell+1)) \\
& \quad=A(\ell-1, \ell+1)-A(\ell, \ell) \geq k
\end{aligned}
$$

whenever $1<\ell<t$. That establishes the Claim for $p=1$. 
Now consider $p>1$, and suppose the Claim holds for $p-1$, i.e.,

$$
A(\ell-(p-1), \ell+1)-A(\ell-(p-1)+1, \ell) \geq k
$$

whenever $p-1<\ell<t$. By H2, the SP at $(\ell-p+1, \ell)$ is at least 0 whenever $p<\ell<t$. Hence

$$
(A(\ell-p+2, \ell)-A(\ell-p+1, \ell))-(A(\ell-p+1, \ell+1)-A(\ell-p, \ell+1)) \geq 0
$$

whenever $p<\ell<t$. Adding the two preceding inequalities, we obtain

$$
A(\ell-p, \ell+1)-A(\ell-p+1, \ell) \geq k
$$

whenever $p<\ell<t$. The induction is complete.

Claim 2: If $m>t$, then the maximal revenue on the $m$ th antidiagonal, and hence the maximal surplus on that antidiagonal,is achieved at the profile $(m-t, t)$ (or $(t, m-t)$ by symmetry). If $2 \leq m \leq t$, then the maximal revenue, and hence the maximal surplus, on the $m$ th antidiagonal is achieved at the profile $(1, m-1)($ or $(m-1,1)$ by symmetry).

Note that $(i, j)$ and $(i-1, j+1)$ lie on the same antidiagonal. Hence Claim 1 tells us that for $m>t$, revenue and surplus increase as we move along the $m$ th antidiagonal in the direction of increasing $j$, reaching a maximum at $j=t$. The second sentence of the Claim holds by symmetry.

Claim 3: The profile $(1, t)$ is efficient if and only if $A(2, t)-A(1, t) \leq k$.

The surplus at $(1, t)$ is $A(1, t)-(t+1) k$. By Claim 2, the profile $(1, t)$ is efficient if and only if this surplus is at least as large as each of the surpluses at $(1,1),(1,2), \ldots$, $(1, t-1)$ and $(2, t),(3, t), \ldots,(t, t)$. If $(1, t)$ is efficient, it is clear that $A(2, t)-$ $A(1, t) \leq k$.

Now consider the converse. Suppose that $A(2, t)-A(1, t) \leq k$. This implies that the surplus at $(1, t)$ is at least as large as the surplus at $(2, t)$. By the DMP property, we have $A(3, t)-A(2, t)<A(2, t)-A(1, t)$, hence $A(3, t)-A(2, t)<k$. Similarly, we have $A(m, t)-A(m-1, t)<k$ for each $m \geq 3$. Therefore for each $m \geq 3$ we have

$$
(A(2, t)-A(1, t))+(A(3, t)-A(2, t))+\cdots+(A(m, t)-A(m-1, t))<(m-1) k .
$$

This inequality simplifies to $A(m, t)-A(1, t)<(m-1) k$. So the surplus at $(1, t)$ is at least as large as each of the surpluses at $(3, t),(4, t), \ldots,(t, t)$.

Next we show that the surplus at $(1, t)$ is at least as large as each of the surpluses at $(1,2), \ldots,(1, t-1)$.

By Claim 1 , we have $A(1, \alpha)-A(2, \alpha-1) \geq k$ for $3 \leq \alpha \leq t$. Then by productivity we have $A(1, \alpha-1) \leq A(2, \alpha-1)$, so that

$$
A(1, \alpha)-A(1, \alpha-1) \geq k \text { for } 3 \leq \alpha \leq t
$$


Thus for each $\alpha$ with $3 \leq \alpha \leq t$, we have

$$
\begin{gathered}
(A(1, \alpha)-A(1, \alpha-1))+(A(1, \alpha+1)-A(1, \alpha)) \\
+\cdots+(A(1, t)-A(1, t-1)) \geq(t-\alpha+1) k
\end{gathered}
$$

which simplifies to $A(1, t)-A(1, \alpha-1) \geq(t-\alpha+1) k$. This implies that the surplus at $(1, t)$ is at least as large as the surpluses at each of $(1,2),(1,3), \ldots,(1, t-1)$.

The last step is to show that the surplus at $(1, t)$ is at least as large as the surplus at $(1,1)$. That is equivalent to

$$
A(1, t)-A(1,1) \geq(t-1) k
$$

By H3, there is some profile $\left(i^{\prime}, j^{\prime}\right)$ with $1<i^{\prime}<j^{\prime}<t$ such that the SP at $\left(i^{\prime}, j^{\prime}\right)$ is at least $k$. Note that $j^{\prime} \geq 3$. We first use $\mathrm{H} 3$ to prove an auxiliary result, namely

(iii) $A\left(i^{\prime}-q, j^{\prime}+1\right)-A\left(i^{\prime}-q+1, j^{\prime}\right) \geq 2 k$ for $q=1,2, \ldots, i^{\prime}-1$.

As in the proof of Claim 1, we perform an induction on $q$. By our assumption on $\left(i^{\prime}, j^{\prime}\right)$ we have

$$
\left(A\left(i^{\prime}+1, j^{\prime}\right)-A\left(i^{\prime}, j^{\prime}\right)\right)-\left(A\left(i^{\prime}, j^{\prime}+1\right)-A\left(i^{\prime}-1, j^{\prime}+1\right)\right) \geq k .
$$

By Claim 1, we have

$$
A\left(i^{\prime}, j^{\prime}+1\right)-A\left(i^{\prime}+1, j^{\prime}\right) \geq k
$$

Adding the above two inequalities, we get

$$
A\left(i^{\prime}-1, j^{\prime}+1\right)-A\left(i^{\prime}, j^{\prime}\right) \geq 2 k
$$

establishing our result for the case $q=1$. For the induction step, suppose that $1<$ $q<i^{\prime}$ and that

$$
A\left(i^{\prime}-(q-1), j^{\prime}+1\right)-A\left(i^{\prime}-(q-1)+1, j^{\prime}\right) \geq 2 k
$$

Then (by H2) the SP at $\left(i^{\prime}-(q-1), j^{\prime}\right)$ is at least 0 , so we have

$$
\begin{aligned}
& \left(A\left(i^{\prime}-(q-1)+1, j^{\prime}\right)-A\left(i^{\prime}-(q-1), j^{\prime}\right)\right)-\left(A\left(i^{\prime}-(q-1), j^{\prime}+1\right)\right. \\
& \left.-A\left(i^{\prime}-(q-1)-1, j^{\prime}+1\right)\right) \geq 0 .
\end{aligned}
$$

Adding the two preceding inequalities, we obtain $A\left(i^{\prime}-q, j^{\prime}+1\right)-A\left(i^{\prime}-q+1, j^{\prime}\right) \geq$ $2 k$, thereby completing the induction and establishing (iii).

For the case $i^{\prime}=2, q=1$, (iii) becomes $A\left(1, j^{\prime}+1\right)-A\left(2, j^{\prime}\right) \geq 2 k$. That, together with productivity, implies

$$
A\left(1, j^{\prime}+1\right)-A\left(1, j^{\prime}\right) \geq 2 k
$$


In view of (i) above we have

$$
\begin{aligned}
& (A(1, t)-A(1, t-1))+(A(1, t-1)-A(1, t-2)) \\
& +\cdots+\left(A\left(1, j^{\prime}+2\right)-A\left(1, j^{\prime}+1\right)\right) \geq\left(t-\left(j^{\prime}+2\right)+1\right) k,
\end{aligned}
$$

which simplifies to $A(1, t)-A\left(1, j^{\prime}+1\right) \geq\left(t-\left(j^{\prime}+2\right)+1\right) k$. Similarly we have

$$
\begin{aligned}
& \left(A\left(1, j^{\prime}\right)-A\left(1, j^{\prime}-1\right)\right)+\left(A\left(1, j^{\prime}-1\right)-A\left(j^{\prime}-2\right)\right)+\cdots+(A(1,4) \\
& -A(1,3))+(A(1,3)-A(1,2)) \geq\left(j^{\prime}-3+1\right) k,
\end{aligned}
$$

which simplifies to $A\left(1, j^{\prime}\right)-A(1,2) \geq\left(j^{\prime}-3+1\right) k$. That, together with productivity, implies

$$
A\left(1, j^{\prime}\right)-A(1,1) \geq\left(j^{\prime}-3+1\right) k .
$$

Using (iii)-(v), we obtain

$$
\begin{aligned}
& \left(A(1, t)-A\left(1, j^{\prime}+1\right)\right)+\left(A\left(j^{\prime}+1\right)-A\left(1, j^{\prime}\right)\right)+\left(A\left(1, j^{\prime}\right)-A(1,1)\right) \\
& \geq\left(t-\left(j^{\prime}+2\right)+1\right) k+2 k+\left(j^{\prime}-3+1\right) k,
\end{aligned}
$$

which simplifies to $A(1, t)-A(1,1) \geq(t-1) k$. So (ii) is established and the proof of Claim 3 is complete.

Since, by $\mathrm{H} 4$, we have $A(2, t)-A(1, t) \leq k$, Claim 3 tells us that $(1, t)$ is indeed efficient.

Part 2. In this part we show that H1, strict productivity, and the DMP property imply that if $(i, j)$, with $i \leq j$, is an equilibrium, then $j \in\{t, t-1,1\}$.

Suppose, to the contrary, that $j=t-u$, for some $u$ satisfying $2 \leq u \leq t-2$. Since $(i, j)$ is an equilibrium we have $A(i+1, j)-A(i, j) \leq 2 k$. That, together with the DMP property, implies

$$
A(j+1, j)-A(j, j) \leq 2 k
$$

By condition $\mathrm{H} 1$, the $\mathrm{SP}$ at $(j, j)$ is at least $k$, i.e.,

$$
(A(j+1, j)-A(j, j))-(A(j, j+1)-A(j-1, j+1)) \geq k .
$$

Now (1) and (2) imply

$$
A(j, j+1)-A(j-1, j+1) \leq k .
$$

That inequality, together with DMP, implies

$$
A(j+2, j+1)-A(j+1, j+1) \leq k .
$$


[Since we assume $t \geq 4$, the strategy $j=t-u$ exists for every $u$ in $[2, t-2]$, and so do $j+1$ and $j+2]$. By H1, the substitutes penalty at $(j+1, j+1)$ is at least $k$, i.e.

$$
[A(j+2, j+1)-A(j+1, j+1)]-[A(j+1, j+2)-A(j, j+2)] \geq k .
$$

Now (3) and (4) imply

$$
A(j+1, j+2)-A(j, j+2) \leq 0
$$

That contradicts strict productivity. Hence $j$ indeed belongs to the set $\{t, t-1,1\}$.

The proof of Theorem $\mathrm{C}$ is complete.

Proof of Theorem $G$ : Let $p(t)$ denote $\sum_{i \in N}\left(t_{i} A(x(t))-c_{i}\left(x_{i}(t)\right)\right.$ (the sum of the players' net payoffs), and let $r(t)$ denote $\left(1-t \sum_{i \in N} r_{i}\right) A(x(t))$ (the residual at $\left.x(t)\right)$. The conclusion of Theorem $\mathrm{G}$ holds if $p(t)$ is increasing and $r(t)$ is decreasing, on some interval containing the number $\epsilon=\left(\sum_{i \in N} r_{i}\right)^{-1}$. As $p$ and $r$ are continuously differentiable, it suffices to show that $p^{\prime}(\epsilon)>0$ and that $r^{\prime}(\epsilon)<0$.

Abbreviating $x(t)$ by $x$ and $x_{j}(t)$ by $x_{j}$, we get

$$
\begin{aligned}
p^{\prime}(t) & =\sum_{i \in N}\left(r_{i} A(x)+t r_{i}\left(\sum_{j \in N} A_{j}(x) x_{j}^{\prime}\right)-c_{i}^{\prime}\left(x_{i}\right) x_{i}^{\prime}\right) \\
& >\sum_{i \in N}\left(t r_{i}\left(\sum_{j \in N} A_{j}(x) x_{j}^{\prime}\right)-c_{i}^{\prime}\left(x_{i}\right) x_{i}^{\prime}\right) \\
& =\sum_{j \in N}\left(t A_{j}(x) x_{j}^{\prime} \sum_{i \in N} r_{i}\right)-\sum_{i \in N} c_{i}^{\prime}\left(x_{i}\right) x_{i}^{\prime} .
\end{aligned}
$$

(Here we are using the facts that each $r_{i} \leq 1$; each $A_{i}$ is positive, by productivity; and each $x_{i}^{\prime}$ is positive by Theorem F). We now evaluate at $t=\epsilon$ and use the fact that $\epsilon \sum_{i \in N} r_{i}=1$ to get

$$
\begin{aligned}
p^{\prime}(\epsilon) & >\sum_{i \in N} x_{i}^{\prime}\left(A_{i}(x)-c_{i}^{\prime}\left(x_{i}\right)\right) \\
& \geq \sum_{i \in N} x_{i}^{\prime} \cdot\left[r_{i} A_{i}(x)-c_{i}^{\prime}\left(x_{i}\right)\right] .
\end{aligned}
$$

Note that the term in square brackets is zero by the first-order conditions that are satisfied by $x$, which is an interior equilibrium. Hence $p^{\prime}(\epsilon)>0$, as claimed. Quite similarly,

$$
r^{\prime}(t)=\left(1-t \sum_{i \in N} r_{i}\right)\left(\sum_{j \in N} A_{j}(x) x_{j}^{\prime}\right)-\left(\sum_{i \in N} r_{i}\right) \cdot A(x(t))
$$


Evaluating at $t=\epsilon$, the first term in parentheses is zero, so we get

$$
r^{\prime}(\epsilon)=\left(-\sum_{i \in N} r_{i}\right) \cdot A(x(t))<0,
$$

since $\sum_{i \in N} r_{i}>0$ and $A>0$. That concludes the proof.

Acknowledgment Courtney was partially supported by NSF VIGRE grant DMS-0602242 to the Department of Mathematics, University of Iowa.

Open Access This article is distributed under the terms of the Creative Commons Attribution Noncommercial License which permits any noncommercial use, distribution, and reproduction in any medium, provided the original author(s) and source are credited.

\section{References}

Arya A, Glover J, Hughes JS (1997) Implementing coordinated team play. J Econ Theory 74:218-232

Che Y-K, Yoo S-W (2001) Optimal incentives for teams. Am Econ Rev 91:525-541

Courtney D, Marschak T (2006) Shirking and squandering in sharing games. Topics in Theoretical Economics, vol 6, issue 1. Berkeley Electronic Press, Berkeley

Holmstrom B (1982) Moral hazard in teams. Bell J Econ 13:324-340

Ishida J (2006) Team incentives under relative perfomance evaluation. J Econ Manag Strategy 15:187-206

Legros P, Matthews S (1993) Efficient and nearly-efficient partnerships. Rev Econ Stud 68:599-611

Levitt S (1995) Optimal incentive schemes when only agents' 'Best' output matters. Rand J Econ 26:744760

Marschak T (2004) Information technology and the organization of firms. J Econ Manag Strategy 13:473516

Mookherjee D (1984) Optimal incentive schemes with many agents. Rev Econ Stud 51:433-446

Monderer D, Shapley S (1996) Potential games. Games Econ Behav 14:124-143

Nandeibam S (2002) Sharing rules in teams. J Econ Theory 107:407-420

Topkis D (1998) Supermodularity and complementarity. Princeton University Press, New Jersey 